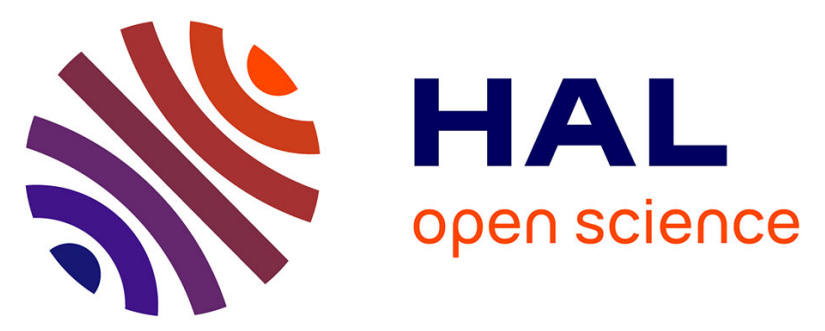

\title{
Construction and identification of a D-Vine model applied to the probability distribution of modal parameters in structural dynamics
}

Sylvian Dubreuil, Michel Salaün, Emmanuel Rodriguez, Frank Petitjean

\section{To cite this version:}

Sylvian Dubreuil, Michel Salaün, Emmanuel Rodriguez, Frank Petitjean. Construction and identification of a D-Vine model applied to the probability distribution of modal parameters in structural dynamics. Mechanical Systems and Signal Processing, 2018, vol. 98, pp. 190-208. 10.1016/j.ymssp.2017.04.024 . hal-01654735

\section{HAL Id: hal-01654735 \\ https://hal.science/hal-01654735}

Submitted on 4 Dec 2017

HAL is a multi-disciplinary open access archive for the deposit and dissemination of scientific research documents, whether they are published or not. The documents may come from teaching and research institutions in France or abroad, or from public or private research centers.
L'archive ouverte pluridisciplinaire HAL, est destinée au dépôt et à la diffusion de documents scientifiques de niveau recherche, publiés ou non, émanant des établissements d'enseignement et de recherche français ou étrangers, des laboratoires publics ou privés. 


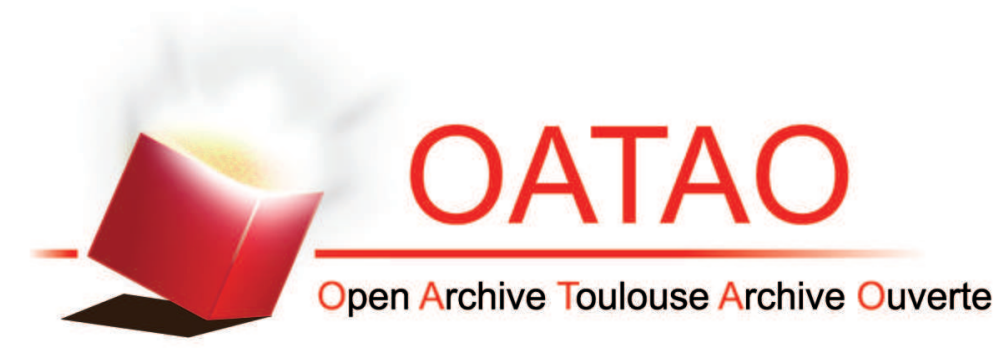

\section{Open Archive Toulouse Archive Ouverte (OATAO)}

OATAO is an open access repository that collects the work of some Toulouse researchers and makes it freely available over the web where possible.

This is an author's version published in: https://oatao.univ-toulouse.fr/18509

Official URL : http://dx.doi.org/10.1016/j.ymssp.2017.04.024

\section{To cite this version :}

Dubreuil, Sylvian and Salaün, Michel and Rodriguez, Emmanuel and Petitjean, Frank Construction and identification of a D-Vine model applied to the probability distribution of modal parameters in structural dynamics. (2018) Mechanical Systems and Signal Processing, vol. 98. pp. 190-208. ISSN 0888-3270

Any correspondence concerning this service should be sent to the repository administrator: tech-oatao@listes-diff.inp-toulouse.fr 


\title{
Construction and identification of a D-Vine model applied to the probability distribution of modal parameters in structural dynamics
}

\author{
S. Dubreuil ${ }^{\mathrm{a}, *}$, M. Salaün ${ }^{\mathrm{b}}$, E. Rodriguez ${ }^{\mathrm{c}}$, F. Petitjean $^{\mathrm{c}}$ \\ a Onera - The French Aerospace Lab, F-31055 Toulouse, France \\ b Institut Clément Ader, CNRS UMR 5312, Université Fédérale Toulouse Midi-Pyrénées, ISAE/INSA/UPS/Mines Albi, 3 rue Caroline Aigle, 31400 Toulouse, France \\ ${ }^{\mathrm{c}}$ Institut Catholique d'Arts et Métiers (ICAM), F-31300 Toulouse, France
}

Keywords:

Probabilistic modal superposition

Random transfer functions

Copula theory

Pair-copula construction

D-Vine

\begin{abstract}
A B S T R A C T
This study investigates the construction and identification of the probability distribution of random modal parameters (natural frequencies and effective parameters) in structural dynamics. As these parameters present various types of dependence structures, the retained approach is based on pair copula construction (PCC). A literature review leads us to choose a $D$-Vine model for the construction of modal parameters probability distributions. Identification of this model is based on likelihood maximization which makes it sensitive to the dimension of the distribution, namely the number of considered modes in our context. To this respect, a mode selection preprocessing step is proposed. It allows the selection of the relevant random modes for a given transfer function. The second point, addressed in this study, concerns the choice of the D-Vine model. Indeed, $D$-Vine model is not uniquely defined. Two strategies are proposed and compared. The first one is based on the context of the study whereas the second one is purely based on statistical considerations. Finally, the proposed approaches are numerically studied and compared with respect to their capabilities, first in the identification of the probability distribution of random modal parameters and second in the estimation of the $99 \%$ quantiles of some transfer functions.
\end{abstract}

\section{Introduction}

Probabilistic approaches are nowadays largely used in structural mechanics to handle quantification and propagation of uncertainties through a mechanical model. A global methodology, introduced for example in [1], is now well established and gives the framework to deal with parametric uncertainties affecting the inputs of a mechanical model. A critical point in this methodology concerns propagation of randomness from the model inputs to its outputs.

In the field of deterministic linear structural dynamics, a common way to solve motion equation is to use modal superposition method. It implies the resolution of an eigenvalue problem and the construction of transfer functions by linear combinations, involving both the eigenvalues and some terms of the eigenvectors, the so-called modal parameters.

\footnotetext{
* Corresponding author.

E-mail addresses: sylvain.dubreuil@onera.fr (S. Dubreuil), michel.salaun@isae.fr (M. Salaün), emmanuel.rodriguez@icam.fr (E. Rodriguez), frank. petitjean@icam.fr (F. Petitjean).
} 
In this context, probabilistic approach leads to a random eigenvalue problem. Determination of the probability distributions of eigenvalues and eigenvectors of random matrices is addressed by random matrix theory [2,3]. Nevertheless, this theory mainly deals with random matrices defined by some specific probability distributions. For example, Gaussian ensembles have been widely studied and many theoretical results describe the statistics of their eigenvalues and eigenvectors. Unfortunately, random matrices encountered in engineering problems do not belong to these sets. In the field of linear stochastic dynamical system, random matrix theory has been applied by [4] where Wishart random matrix model is studied and by $[5,6]$ where a non parametric random matrix model is constructed by the use of maximum entropy principle.

A large class of approximation methods has also been successfully applied to this problem. One of the first is based on Taylor series or perturbation method [7]. The main idea is to use first or second order Taylor series expansions of the eigenvalues and eigenvectors in terms of the random inputs. Polynomial chaos expansion [8,9], which is based on the decomposition of second order random variables into a polynomial expansion, has also been applied to random eigenvalue problems. For example in [10], a comparison between polynomial chaos representation and perturbation method is provided, concluding that the former performed better than the latter except if the variability of random inputs is small. In [11] an asymptotic approach is proposed which leads to an expression of the joint probability density function of the eigenvalues. According to the presented results, this approximation approach outperforms second order perturbation approach. However, the random effective parameters are not considered in this work. Polynomial chaos expansion has also been applied to various structural dynamics application (see [12] for example). Another method, based on a dimensional decomposition of a random variable [13], is applied to the random eigenvalues problem in [14]. Finally, metamodels approaches can also be applied such as surface response [15]. It should also be noted that approximation methods have been applied to the resolution of random vibration analysis out of the framework of modal superposition. For example, proper generalized decomposition approximation is used in [16] for the problem of uncertainty in structural dynamics, leading to a reduced order model based on deterministic basis. As these methods are based on functional approximations, their computational cost increase with the number of random inputs which is their main drawback.

One can also note that simulation based methods are relevant in this context as they are not sensitive to the stochastic dimensionality of the problem and are usually easy to implement on industrial models. In theory, direct Monte Carlo Simulation (MCS) method could be used to solve any random eigenvalues problem. But the numerical cost, it involves, becomes unrealistic when one deals with large industrial models. Consequently, research activities on this field are mainly devoted to increasing the computation efficiency of the resolution of one realization of the random eigenvalues problem. For example, $[17,18]$ propose to use subspace iteration method and selection of smart starting vector to increase the efficiency of the eigenvalues problem solver.

The method, we propose in the following, is based on the identification of the probability distribution of the modal parameters involved in a given random transfer function. It should be noted that the identified probability distribution can then be used to perform various statistical analysis of the transfer function at low computational cost (as the expression of the transfer function with respect to the modal parameters is analytic). Especially, one can easily study the random transfer function for a wide range of input excitation (external force or imposed motion of the junction). The keystone is then the construction and identification of large dimension probability distribution. A relevant way to achieve this goal is proposed in $[19,20]$. This method, called pair copula construction (PCC) of joint probability distribution, is based on identification of bivariate copula [21]. We propose to apply this methodology to the identification of the probability distribution of modal parameters. Objectives of this study are mainly to adapt this identification method to the context of structural dynamics and to present the interests of this approach.

The next section of this paper gives some details about the problem that we are interested in: Identification of the modal parameters probability distribution and computation of random transfer function by modal superposition. The third section recalls basics on PCC. The fourth shows how to use PCC in identification of modal parameters probability distribution and highlights specific difficulties. Section five gives applications of the proposed methodology. Finally a concluding part recalls and discusses the results.

\section{Modal superposition method and random eigenvalue problem}

\subsection{Deterministic case}

We consider the vibratory motion of an elastic body excited either by an external force or by a junction displacement, in the low frequency range. The structure is assumed to be discretized by the finite element method. Using the notations introduced in [22], the motion equation reads

$$
\left(-\omega^{2}\left[\begin{array}{ll}
\underline{\mathbf{M}}_{i i} & \underline{\mathbf{M}}_{i l} \\
\underline{\mathbf{M}}_{l i} & \underline{\mathbf{M}}_{l l}
\end{array}\right]+\mathrm{j} \omega\left[\begin{array}{ll}
\underline{\mathbf{C}}_{i i} & \underline{\mathbf{C}}_{i l} \\
\underline{\mathbf{C}}_{l i} & \underline{\mathbf{C}}_{l l}
\end{array}\right]+\left[\begin{array}{ll}
\underline{\mathbf{K}}_{i i} & \underline{\mathbf{K}}_{i l} \\
\underline{\mathbf{K}}_{l i} & \underline{\mathbf{K}}_{l l}
\end{array}\right]\right)\left\{\begin{array}{l}
\underline{\mathbf{X}}_{i}(\omega) \\
\underline{\mathbf{X}}_{l}(\omega)
\end{array}\right\}=\left\{\begin{array}{l}
\underline{\mathbf{F}}_{i}(\omega) \\
\underline{\mathbf{F}}_{l}(\omega)
\end{array}\right\}
$$

where $\mathrm{j}^{2}=-1$. A matrix $\mathbf{X}_{i l}$ denotes a matrix of size $\left(n_{i}, n_{l}\right)$, where $n_{i}$ is the number of internal DOF (degrees of freedom), indexed by $i$, and $n_{l}$ the number of junction DOF, indexed by $l$. Moreover, with these notations, $\mathbf{X}_{l i}$ is the transposed matrix of $\mathbf{X}_{i l}$ and $\mathbf{X}_{i i}$ is symmetric. $[\underline{\mathbf{M}}],[\underline{\mathbf{K}}]$ and $[\underline{\mathbf{C}}]$ are respectively the mass, stiffness and damping matrices of the structure, calculated for the mean values of input parameters. $\underline{\mathbf{X}}_{i}$ is the vector of internal DOF, $\underline{\mathbf{X}}_{l}$ the vector of junction DOF, $\underline{\mathbf{F}}_{i}$ the vector of 
external forces and $\underline{\mathbf{F}}_{l}$ the vector of reaction forces. Finally, an underline subscribe indicates an extracted component (for example, $\mathbf{X}_{i i}$ refers to a line of matrix $\mathbf{X}_{i i}$.

In low frequency range, modal superposition is classically used to solve Eq. (1). It consists in a projection into a basis formed by the $n_{k}$ first eigenvectors of $\underline{\mathbf{K}}_{i i}$ with respect to $\underline{\mathbf{M}}_{i i}$. Let us set $\underline{\lambda_{k}}$ and $\underline{\varphi}_{\underline{i} \underline{k}}$, with $\underline{k} \in\left[1, n_{k}\right]$, the corresponding eigenvalues and eigenvectors (eigenvectors are classically normalized with respect to the mass matrix). The modal superposition consists in the projection

$$
\left\{\begin{array}{l}
\underline{\mathbf{X}}_{i} \\
\underline{\mathbf{X}}_{l}
\end{array}\right\}=\left[\begin{array}{cc}
\underline{\varphi}_{i k} & \underline{\psi}_{i l} \\
\overline{\mathbf{0}}_{l k} & \mathbf{I}_{l l}
\end{array}\right]\left\{\begin{array}{l}
\underline{\mathbf{q}}_{k} \\
\underline{\mathbf{X}}_{l}
\end{array}\right\}
$$

where $\underline{\psi}_{i l}$ are the static junction shapes. Then, solution of Eq. (1) is expressed thanks to transfer functions

$$
\left\{\begin{array}{c}
\underline{\mathbf{X}}_{i}(\omega) \\
\underline{\mathbf{F}}_{l}(\omega)
\end{array}\right\}=\left[\begin{array}{cc}
\underline{\mathbf{G}}_{i i}(\omega) & \underline{\mathbf{T}}_{i l}(\omega) \\
-\underline{\mathbf{T}}_{l i}(\omega) & \underline{\mathbf{S}}_{l l}(\omega)
\end{array}\right]\left\{\begin{array}{c}
\underline{\mathbf{F}}_{i}(\omega) \\
\underline{\mathbf{X}}_{l}(\omega)
\end{array}\right\}
$$

where $[\underline{\mathbf{G}}]_{i i}(\omega)$ is the dynamic flexibility, $[\underline{\mathbf{T}}]_{i l}(\omega)$ the dynamic transmissibility and $[\underline{\mathbf{S}}]_{l l}(\omega)$ the dynamic stiffness. Although the proposed approach may be applied to any of these three transfer functions, the paper is focused on the dynamic transmissibility defined by,

$$
\left[\underline{\mathbf{T}}_{i l}(\omega)=[\underline{\boldsymbol{\psi}}]_{i l}+\omega^{2} \sum_{\underline{\underline{k}}=1}^{n_{i}} \underline{H}_{\underline{k}}(\omega) \underline{\boldsymbol{\varphi}_{i} \underline{\underline{\mathbf{L}}} \underline{\underline{k}} l},\right.
$$

where $\underline{H}_{k}$ is the dynamic amplification factor of mode $\underline{k}$

$$
\underline{H_{k}}(\omega)=\frac{1}{-\omega^{2}+\underline{\lambda_{k}}+2 \mathrm{j} \omega \underline{\xi}_{\underline{k}} \sqrt{\underline{\lambda_{k}}}}
$$

where the modal damping ratio is $2 \xi_{\underline{k}} \sqrt{\underline{\underline{\lambda}} \underline{k}}$ for mode $\underline{k}$. The other terms are given by

$$
\underline{\mathbf{L}}_{k l}=\left[\underline{\boldsymbol{\varphi}}_{k i}\right]\left([\underline{\mathbf{M}}]_{i i}[\underline{\underline{\psi}}]_{i l}+[\underline{\mathbf{M}}]_{i l}\right)
$$

Eq. (3) shows that this transfer function results of a sum of products between the amplification factor of mode $\underline{k}$ and another term depending on mode $\underline{k}$, called effective parameter and linked to the eigenvector (the same remark can be done for the two other transfer functions). More precisely, for the dynamic transmissibility, the effective parameters are called effective transmissibilities and are equal to $\left[\tilde{\mathbf{T}}_{i l, \underline{k}}=\underline{\boldsymbol{\varphi}}_{\underline{i k} \underline{\underline{\mathbf{L}}} \underline{\underline{k} l}}\right.$ for each mode $\underline{k}$.

After this presentation of the deterministic problem, the next part introduces parametric uncertainties and describes the stochastic problem.

\subsection{Parametric model for random uncertainties}

In the following it is assumed that the uncertain input parameters (material and geometric properties) of the finite element model can be modeled by a random vector and that the probability distribution of this random vector is known. Consequently, the responses of the model are also random quantities. As mentioned previously only the dynamic transmissibility is studied in the following and more precisely its modulus. Thus, the paper focuses on the behavior of the random process (indexed by $\omega$ ),

$$
\left|T_{a c}(\omega)\right|=\left|\underline{\psi_{a c}}+\omega^{2} \sum_{k=1}^{n_{k}} H_{k}\left(\omega, \Lambda_{k}\right) \widetilde{T}_{a c, k}\right|
$$

where $a$ denotes an internal DOF, $c$ denotes a junction DOF and $\Lambda_{k}, \widetilde{T}_{a c, k}$ are the $n_{k}$ random eigenvalues and random effective parameters respectively (random modal parameters). Note that, to simplify notations, subscribes are no longer underlined when they denote components and that no confusion with previous notation is possible as only one dimensional random transfer functions are considered in the following.

In order to efficiently investigate the probability distribution of $\left|T_{a c}(\omega)\right|$ we propose to identify the joint probability distribution of the random vector $\mathbf{V}_{T}=\left\{\Lambda_{k}, \widetilde{T}_{a c, k}, k \in\left[1, n_{k}\right]\right\}$. Indeed, following equations Eqs. (3)-(5) one can note that the random transfer function has a closed form expression with respect to the random vector $\mathbf{V}_{T}$. Hence, once the probability of $\mathbf{V}_{T}$ is identified, one can perform various statistical analyses of the random transfer function by Monte Carlo approach at a very low computational cost. Moreover, one can note that it also allows to study the random transfer function with respect to various input excitation $\left(\underline{\mathbf{F}}_{i}(\omega)\right.$ and $\left.\underline{\mathbf{X}}_{l}(\omega)\right)$, various damping coefficients $\xi_{k}$ and for several pulsations $\omega$. The main contributions of the paper are thus to present, first, an identification method of the probability distribution of $\mathbf{V}_{T}$ based on PCC and, second, the possible benefits of this approach in the computation of the random transfer function high quantiles compared to direct Monte Carlo approach. It should be noted that this possible benefit is expected due to the behavior of the probability density function of the dynamic amplification factor of a single DOF system subjected to a random eigen- 
value. Indeed, in $[23,24]$ a detailed study of this bounded probability density function shows that, in the vicinity of the square root of the mean eigenvalue, it tends to infinity at its upper bound (which is a deterministic value with a tractable analytical expression in the single DOF case). Hence, the estimation of the upper quantiles is strongly influenced by the way the probability density function tends to infinity (and which depends on the expression of the dynamic amplification factor given by Eq. (4)) and slightly by the probability distribution of the random eigenvalue. Hence, one can roughly estimate the probability distribution of the eigenvalue and then use it in the numerical integration of the expression of the probability distribution of the dynamic amplification factor in order to get an accurate estimation of the upper quantiles.

In the framework of multi DOF systems, the analytic expression of the probability distribution of the transfer function Eq. (5) does not have any closed form expression. However one can rely on Monte Carlo estimation to compute upper quantiles. Moreover, based on the remark about single DOF system one may expect these upper quantiles to be only slightly influenced by the probability distribution of the modal parameters. Hence, the idea we propose in this paper is to identify the joint probability density function of the modal parameters based on a small identification sample (as the creation of this sample involves the resolution of costly eigenvalue problems) and then, to use it to estimate the probability distribution of the transfer function based on a larger sample (but with a negligible numerical cost as the expression of the transfer function is analytic with respect to the modal parameters). Numerical examples provided in Section 5 will investigate the accuracy of the proposed approach compared to direct Monte Carlo approach.

Remark on random eigenvalues realization: In the field of random linear structural dynamics, a large literature is devoted to the study of mode degenerative phenomena [25] (such as mode crossing and mode veering). These phenomena have been observed and studied not only numerically but also experimentally for example in [26]. A major difficulty is then the association of the eigenvalues realization to the corresponding random eigenvalues. Various procedures have been proposed (see [27-29] and [30,31] for meta-model approaches) to perform this association step (sometimes called mode tracking) and it should be emphasized that our approach is not dependent of the retained procedure. In the following numerical examples, eigenvalues are associated with respect to their rank in ascending order. This methodology is retained for its simplicity. Moreover, application examples will show that modes degenerative phenomena can be interpreted in terms of dependence structure of the random effective parameters. More precisely, the numerical example introduced in Section 5.2, presents an example of mode crossing between two close modes with very different effective parameters. Interpretation of dependence structure between modal parameters for identification of more general mode degenerative phenomena must be seen as a perspective of this work and need a dedicated study.

\section{Pair copula construction of joint probability distribution}

\subsection{Bivariate copula}

This part is devoted to a short presentation of copula theory, emphasizing the two dimensional case. Basics on copula theory and construction of joint probability distributions can be found in [21,32]. For application in engineering and more precisely in reliability, we refer to Lebrun and Dutfoy [33-35]. First of all, let us introduce the definition of a copula [21].

Bivariate copula. A bivariate copula is a function $C$ defined on $[0,1]^{2}$ and such as

1. $\forall(x, y) \in[0,1]^{2}, C(x, 0)=C(0, y)=0$

2. $\forall(x, y) \in[0,1]^{2}, C(x, 1)=x$ and $C(1, y)=y$

3. $\forall\left(x_{1}, y_{1}\right) \in[0,1]^{2},\left(x_{2}, y_{2}\right) \in[0,1]^{2}$, such as $0 \leqslant x_{1} \leqslant x_{2} \leqslant 1$ and $0 \leqslant y_{1} \leqslant y_{2} \leqslant 1$

$$
C\left(x_{1}, y_{1}\right)-C\left(x_{1}, y_{2}\right)-C\left(x_{2}, y_{1}\right)+C\left(x_{2}, y_{2}\right) \geqslant 0
$$

Let $\mathbf{X}$ be a two dimensional continuous random variable with cumulative distribution function (CDF) $F_{\mathbf{X}}\left(x_{1}, x_{2}\right)$, probability distribution function (PDF) $p_{\mathbf{X}}\left(x_{1}, x_{2}\right)$, marginal CDF $F_{X_{1}}\left(x_{1}\right)$ and $F_{X_{2}}\left(x_{2}\right)$ and marginal PDF $p_{X_{1}}\left(x_{1}\right)$ and $p_{X_{2}}\left(x_{2}\right)$, then there exists a copula $C$ such that (see [36]):

$$
p_{\mathbf{X}}\left(x_{1}, x_{2}\right)=c\left(F_{X_{1}}\left(x_{1}\right), F_{X_{2}}\left(x_{2}\right)\right) p_{X_{1}}\left(x_{1}\right) p_{X_{2}}\left(x_{2}\right) .
$$

where

$$
c\left(u_{1}, u_{2}\right)=\frac{\partial^{2} C\left(u_{1}, u_{2}\right)}{\partial u_{1} \partial u_{2}}
$$

Eq. (6) shows that the copula handles the dependence structure between the two random variables $X_{1}$ and $X_{2}$. This formalism allows to easily specify the dependence structure independently of the marginals. The two most famous parametric copulas families are elliptical copulas and the archimedean copulas [37]. Let us precise that copulas can be generalized to dimension $n$. Nevertheless, using a $n$ variate copula implies the same dependence structure between all pairs of variables. In order to construct a $n$ variate probability distribution with different dependence structures, the next part introduces pair copula construction of multivariate probability distributions. 


\subsection{Pair construction and Vine}

First of all, let us recall that a PDF of dimension $n$ can be factorized by using conditional PDF. Using copulas formalism this implies that any PDF of dimension $n$ can be written as the product of $n$ marginal PDF by $n(n-1) / 2$ bivariate copulas. Proof of this result is given in [38]. To illustrate it, we consider $\mathbf{X}=\left(X_{1}, X_{2}, X_{3}, X_{4}\right)$ a continuous random variable of dimension $n=4$. We call $p_{\mathbf{X}}$ its PDF and $p_{X_{i}}, i=1, \ldots, 4$ its marginal PDF. Then, $p_{\mathbf{X}}$ can be factorized as

$$
p_{\mathbf{X}}\left(x_{1}, x_{2}, x_{3}, x_{4}\right)=p_{X_{1}}\left(x_{1}\right) p_{X_{2} \mid X_{1}=x_{1}}\left(x_{2}\right) p_{X_{3} \mid X_{1}=x_{1}, X_{2}=x_{2}}\left(x_{3}\right) p_{X_{4} \mid X_{1}=x_{1}, X_{2}=x_{2}, X_{3}=x_{3}}\left(x_{4}\right)
$$

Then, noticing that

$$
p_{X_{2} \mid X_{1}=x_{1}}\left(x_{2}\right)=\frac{p_{X_{1}, X_{2}}\left(x_{1}, x_{2}\right)}{p_{X_{1}}\left(x_{1}\right)}=c_{1,2}\left(F_{X_{1}}\left(x_{1}\right), F_{X_{2}}\left(x_{2}\right)\right) p_{X_{2}}\left(x_{2}\right)
$$

and

$$
\begin{aligned}
p_{X_{3} \mid X_{1}=x_{1}, X_{2}=x_{2}}\left(x_{3}\right) & =\frac{p_{X_{1}, X_{2}, X_{3}\left(x_{1}, x_{2}, X_{3}\right)}}{p_{X_{1}, X_{2}}\left(x_{1}, x_{2}\right)} \\
& =\frac{p_{X_{1}, X_{3} \mid X_{2}=x_{2}\left(x_{1}, x_{3}\right) p_{X_{2}}\left(X_{2}\right)}}{p_{X_{1} \mid X_{2}=x_{2}\left(x_{1}\right)}} \\
& =c_{1,3 \mid 2}\left(F_{X_{1} \mid X_{2}=x_{2}}\left(x_{1}\right), F_{X_{3} \mid X_{2}=x_{2}}\left(x_{3}\right)\right) p_{X_{3} \mid X_{2}=x_{2}}\left(x_{3}\right) \\
& =c_{1,3 \mid 2}\left(F_{X_{1} \mid X_{2}=x_{2}}\left(x_{1}\right), F_{X_{3} \mid X_{2}=x_{2}}\left(x_{3}\right)\right) c_{2,3}\left(F_{X_{2}}\left(x_{2}\right), F_{X_{3}}\left(x_{3}\right)\right) p_{X_{3}}\left(x_{3}\right)
\end{aligned}
$$

where $c_{i, j}$ is the derivative of the copula $C_{i, j}$ modeling the dependence structure between $X_{i}$ and $X_{j}$ and $C_{i, j k}$ is the copula modeling the dependence structure between $X_{i} \mid X_{k}=x_{k}$ and $X_{j} \mid X_{k}=x_{k}$. It is clear that each term of decomposition Eq. (7) can be expressed as the product of bivariate copulas and one marginal PDF, leading to

$$
\begin{aligned}
p\left(x_{1}, x_{2}, x_{3}, x_{4}\right)= & p_{X_{1}}\left(x_{1}\right) p_{X_{2}}\left(x_{2}\right) p_{X_{3}}\left(x_{3}\right) p_{X_{4}}\left(x_{4}\right) \\
& c_{1,2}\left(F_{X_{1}}\left(x_{1}\right), F_{X_{2}}\left(x_{2}\right)\right) c_{2,3}\left(F_{X_{2}}\left(x_{2}\right), F_{X_{3}}\left(x_{3}\right)\right) c_{3,4}\left(F_{X_{3}}\left(x_{3}\right), F_{X_{4}}\left(x_{4}\right)\right) \\
& c_{1,3 \mid 2}\left(F_{X_{1} \mid X_{2}=x_{2}}\left(x_{1}\right), F_{X_{3} \mid X_{2}=x_{2}}\left(x_{3}\right)\right) c_{2,4 \mid 3}\left(F_{X_{2} \mid X_{3}=x_{3}}\left(x_{2}\right), F_{X_{4} \mid X_{2}=x_{2}}\left(x_{4}\right)\right) \\
& c_{1,4 \mid 2,3}\left(F_{X_{1} \mid X_{2}=x_{2}, X_{3}=x_{3}}\left(x_{1}\right), F_{X_{4} \mid X_{2}=x_{2}, X_{3}=x_{3}}\left(x_{4}\right)\right)
\end{aligned}
$$

Let us remark that decomposition Eq. (8) is not unique as the order in the choice of conditional variables can be changed. In order to organize the various possibilities, a model, called Vine decomposition, is introduced in [19]. In the following, we only present the $D$-Vines (drawable vines), which reads

$$
p_{\mathbf{x}}\left(x_{1}, \ldots, x_{n}\right)=\left(\prod_{j=1}^{n-1} \prod_{i=1}^{n-j} c_{i,(i+j)(i+1), \ldots,(i+j-1)}\right) \prod_{k=1}^{n} p_{X_{k}}\left(x_{k}\right)
$$

where

$$
c_{i, j \mid i_{1}, \ldots, i_{k}}:=c_{i, j \mid i_{1}, \ldots, i_{k}}\left(F_{X_{i} \mid X_{i_{1}}=x_{i_{1}}, \ldots, X_{i_{k}}=x_{i_{k}}}\left(x_{i}\right), F_{X_{j} \mid X_{i_{1}}=x_{i_{1}}, \ldots, X_{i_{k}}=x_{i_{k}}}\left(x_{j}\right)\right) .
$$

For a better understanding of this expressions, Fig. 1 gives the graphical representation of a $D$-Vine in dimension $n=4$. This decomposition involves $n-1$ levels or trees, while each level, say $L_{l}$, has $n-l+1$ nodes standing for the variables of the level. Hence, it counts $n(n-1) / 2$ bivariate copulas that must be constructed to describe the dependence structure of the whole random vector.

Finally, one can remark that this decomposition is not uniquely defined as the order of the variables at the first level can be exchanged. Section 4.2 will discuss these points.

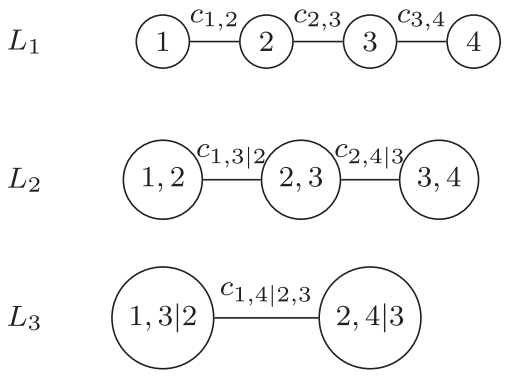

Fig. 1. Example of $D$-Vine decomposition with $n=4$. 
Remark: Among the various Vine decompositions introduced in [19], we retained the D-Vine as it gives the same weight to all the variables of the first level (correlations are expressed in line). Contrarily, when a variable should be considered as a central node, the $C$-Vine model could be used, in which all correlation are expressed with respect to this central node.

\subsection{Identification}

\subsubsection{Simplified $P C C$ and conditional cumulative distribution function}

Eq. (9) and Fig. 1 show that, except for the first level, the bi-dimensional copulas are conditional and that the number of conditioning variables increases by one at each level. In order to identify these copulas, it is generally assumed that these conditional copulas are independent of the value of the conditioning variables. This assumption leads to simplified PCC. Effect of this simplification has been studied in [39], concluding that, in many cases, a PCC is very well approximated by such a simplified version. Moreover, this assumption is also discussed in [40] and the authors show that multivariate normal and Student-t distributions are simplified PCC. Based on these conclusions, simplified PCC will be used in the following.

This assumption allows to create samples of conditional CDF recursively, from the results of the previous level in the Vine decomposition (see the expression of the conditional CDF with respect to the copulas of the previous levels in [32]).

\subsubsection{Stepwise semi parametric estimation}

Identification of a PCC model involves 3 steps.

1. Choice of a Vine decomposition (D-Vine in our context).

2. Choice of the variables order at the first level.

3. Identification of the copulas of the retained model.

Steps 1 and 2 depend on the application and are the original development of this paper; it is discussed in Section 4.2. Step 3 needs two substeps which are, for each bivariate copula, the choice of the parametric copula and the determination of its parameters values.

The choice of each bi-dimensional copula among a family of candidate copulas is performed by the use of Akaike information criterion (AIC) [41].

Parameters identification is achieved by maximization of the log likelihood function (see expressions in [38]). More precisely, we rely on the stepwise semi parametric (SSP) estimation introduced in [38]. It consists in an optimization of the likelihood function at each level of the D-Vine which makes it numerically efficient compared to the optimization of the whole likelihood function (stepwise optimization). Moreover, this approach is called semi parametric as it uses non parametric estimation of the marginal PDF [42] (in practice we use kernel smoothing with standard normal kernel).

Finally, this estimation procedure has been implemented by [43] into a $R$ package used in the following numerical examples.

Remarks:

- The identification of each bivariate copula is parametric and thus suffers from the classical drawbacks of parametric identification. First, if none of the parametric copula available in the family of candidate copulas is suited to represent the data, then one can expect a poor identification. However, the family of candidate copulas implemented in the R package by [43] provides various copulas allowing to represent many shapes of dependence structure. Second, the size of the sample used to identify the copulas strongly influences the accuracy of the identification. In the following numerical examples, the accuracy with respect to this parameter will be systematically studied.

- Accuracy of the SSP compared to the optimization of the whole likelihood function is discussed in [44] and it appears that SSP reduces the asymptotic efficiency of parameters estimators but, in most of the cases, "the performance of the SSP estimator is overall rather good compared to SP" [44]. Concerning application in structural dynamics, a comparison is also provided in [24] concluding that the approximation introduced by the SSP is negligible.

\section{Adaptation to random eigenvalue problem in structural dynamics}

\subsection{Modes selection}

\subsubsection{Deterministic truncation}

Section 2.1 introduced the deterministic modal superposition and the basis $\varphi_{i k}$ made by the $n_{k}$ first eigenvectors of the model, but did not discuss the choice of $n_{k}$. In practice, modal superposition method is interesting in the case where a small subset of modes $n_{k}$ can represent the dynamic behavior of the structure on a given frequency range of the form $\left.] 0, B_{u p}\right] \mathrm{Hz}$. Due to the behavior of the amplification factor, a common practice is to keep all modes that have a natural frequency less than $C B_{u p}$, where $C>1$ is a constant often chosen equal to $\sqrt{2}$.

It is assumed that the same number of modes is kept for the probabilistic approach, hence, the random vectors $\mathbf{V}_{T}$ is of dimension $n=2 n_{k}$. Even if $n_{k}$ is very low compared to the number of DOF, it can be equal to a few dozens. Then, it does not 
seem reasonable to directly apply PCC for the construction of such a large random vector. To this respect, the next part presents a mode selection method.

\subsubsection{Forward selection of relevant modes}

Here is presented a simple way to select a relevant subset of modes for a given random transfer function $T_{a c}(\omega)$. For this, let us introduce $T_{a c}^{n_{i}}(\omega)$ the approximation of $T_{a c}(\omega)$, constructed by using a subset of $n_{i}$ modes. In order to select a relevant subset of size $n_{i}$, the following approximation criterion is used

$$
\epsilon^{n_{i}}=E\left[\int_{0}^{2 \pi B_{u p}}\left(\left|T_{a c}(\omega)\right|-\left|T_{a c}^{n_{i}}(\omega)\right|\right)^{2} d \omega\right]
$$

where $E$ stands for the mathematical expectation.

This criterion is estimated from a sample of size $N_{s}$. The pulsation range of interest is discretized into $N_{p}$ steps of same size $\Delta \omega$. This leads to an estimator of Eq. (10), which reads

$$
\hat{\epsilon}^{n_{i}}=\frac{\Delta \omega}{N_{s}} \sum_{r=1}^{N_{s}}\left(\sum_{k=1}^{N_{p}}\left(\left|t_{a c}^{(r)}\left(\omega_{k}\right)\right|-\left|t_{a c}^{n_{i}(r)}\left(\omega_{k}\right)\right|\right)^{2}\right)
$$

where $t_{a c}^{(r)}$ and $t_{a c}^{n_{i c}(r)}$ are realizations $(r)$ of the random transfer functions $T_{a c}$ and $T_{a c}^{n_{i}}(\omega)$ respectively.

A classical forward selection algorithm is set up to build the subset of size $n_{i}$. The set of candidates modes at the $i$ th step of the algorithm is denoted by $\mathcal{A}^{i}$ ( $\mathcal{A}^{0}$ is composed of the $n_{k}$ modes). The following algorithm is applied,

1. Evaluate $\hat{\epsilon}^{n_{0}}$.

2. For $i=0: n_{k}$

(a) For each mode $m$ in $\mathcal{A}^{i}$,

- Compute $t_{a c}^{n_{i}+m,(r)}\left(\omega_{k}\right)=t_{a c}^{n_{i}(r)}\left(\omega_{k}\right)+\tilde{t}_{a c, m}^{(r)} H\left(\lambda_{m}^{(r)}, \omega_{k}\right), \forall r \in 1, \ldots, N_{s}$, where $\tilde{t}_{a c, m}^{(r)}$ and $\lambda_{m}^{(r)}$ are respectively realization $(r)$ of the effective parameter and of the eigenvalue of mode $m$.

- Compute $\hat{\epsilon}^{n_{i}+m}$

(b) Find $m^{\star}$ such that $\hat{\epsilon}^{n_{i}+m^{\star}}=\min _{m \in\left[1, n_{k}-n_{i}\right]}\left(\hat{\epsilon}^{n_{i}+m}\right)$

(c) Set $t_{a c}^{n_{i+1},(r)}\left(\omega_{k}\right)=t_{a c}^{n_{i}(r)}\left(\omega_{k}\right)+\tilde{t}_{a c, m^{\star}}^{(r)} H\left(\lambda_{m^{\star}}^{(r)}, \omega_{k}\right), \forall r \in 1, \ldots, N_{s}$.

(d) Mode $m^{\star}$ is removed from the candidate modes $\mathcal{A}^{i}$ to form the new set $\mathcal{A}^{i+1}$

3. Choose a set of $n_{i}$ modes accurate enough according to $\frac{\hat{\epsilon}_{i}^{n_{i}}}{\hat{\epsilon}^{0}}<\epsilon_{o b j}$. Influence of the numerical value of $\epsilon_{o b j}$ will be discussed in the numerical examples (Section 5).

This method allows to select only the relevant modes to be part of vector $\mathbf{V}_{T}$. The modal parameters of the non selected modes are set to their mean values. Section 5 will show that, in practical applications, a small subset $n_{i}$ of modes allows to get an accurate representation of the studied random transfer function. Numerical convergence study of this criterion with respect to the size $N_{s}$ of the sample will also be discussed.

\subsection{Variables ordering at the first level of the D-Vine}

Concerning variables ordering at the first level, for small dimensions $(n=3,4)$, [38] suggests to try all possibilities and to choose the best one, according to a given criterion (likelihood for example). For higher dimensions, the number of possible decompositions makes this approach infeasible and authors claim that "one should instead consider which bivariate relationships that are most important to model correctly, and let this determine which decomposition(s) to estimate" [38]. In the following two strategies are compared, each one corresponding to a vision of what is important to model correctly.

To introduce these two strategies, one can first note that, in a D-Vine decomposition, each variable is involved in two bivariate relations, except the first and the last ones that are only involved in one relation. The two proposed strategies are thus defined as follow.

- $S_{1}$ : The order of variables is chosen to model the bivariate relationship between the eigenvalue and the effective parameter of each mode. So, each variable is involved in one bivariate relation $\left(\Lambda_{k}, \widetilde{T}_{a c, k}\right)$. Concerning the second bivariate relation, we propose to model the bivariate relationship between two successive modes. It leads to the following order

$$
\Lambda_{1}, \widetilde{T}_{a c, 1}, \Lambda_{2}, \widetilde{T}_{a c, 2}, \Lambda_{3}, \widetilde{T}_{a c, 3}, \ldots, \Lambda_{n_{i}}, \widetilde{T}_{a c, n_{i}}
$$

In this case, a bivariate relation is important to model correctly, first, if it links modal parameters of a given mode and, second, if it links close modes (with respect to their mean natural frequencies). 
- $S_{2}$ : The bivariate relationships are chosen to be part of the first level according to the strength of their correlation measure (in practice Kendall's $\tau$ [45] is retained as correlation measure). Contrary to $S_{1}$, this strategy does not take into account the context of the study and adopts a purely statistical vision. Practically, Kendall's $\tau$ matrix of vector $\mathbf{V}_{T}$ is estimated from the sample of size $N_{s}$ and a greedy algorithm is set up to choose the variables ordering at the first level. It proceeds in the following way.

1. Compute an estimation of Kendall's $\tau$ matrix from the sample of $\mathbf{V}_{T}$. Let $\tau_{i, j}$ be the estimation of Kendall's $\tau$ coefficient between components $V_{T_{i}}$ and $V_{T_{j}}$ of $\mathbf{V}_{T}$.

2. While $\operatorname{card}\left\{L_{1}\right\}<4 n_{i}-2$,

(a) Find $\tau_{i^{\star}, j^{\star}}=\max \left(\tau_{i, j}\right)$ for $i<j \leqslant 2 n_{i}$.

(b) Check if the bivariate relation between $V_{T_{i^{\star}}}$ and $V_{T_{j^{\star}}}$ is compatible with the bivariate relations, already selected for the first level of decomposition. In practice, a bivariate relationship may be incompatible for two reasons: if one of the two variables is already engaged in two bivariate relationships, or if both variables are engaged in one bivariate relationship and already linked by other bivariate relationships. An illustration of these compatibility conditions is presented in the following remark.

- If relation is incompatible, then set $\tau_{i^{\star} j^{\star}}=0$ and restart from (a).

- If relation is compatible, then $V_{T_{i^{\star}}}$ and $V_{T_{j^{\star}}}$ are add to $L_{1}$ and the bivariate relation is add to the first level. Set $\tau_{i^{\star}, j^{\star}}=0$ and restart from $(a)$.

At the last iteration, the ordering of the $2 n_{i}$ variables for the $D$-Vine first level is obtained.

\section{Remark: illustration of compatibility conditions.}

We assume that the state of the first level of the decomposition at $k$ th iteration is

$$
V_{T_{5}}-V_{T_{9}}-V_{T_{11}}-V_{T_{1}} \quad V_{T_{3}}-V_{T_{4}}-V_{T_{15}}
$$

Then the following examples illustrates compatibility conditions.

- $V_{T_{i^{\star}}}=V_{T_{9}}, V_{T_{j^{\star}}}=V_{T_{3}} \Rightarrow$ incompatible as $V_{T_{9}}$ is already engaged in two relationships.

- $V_{T_{i^{\star}}}=V_{T_{5}}, V_{T_{j^{\star}}}=V_{T_{1}} \Rightarrow$ incompatible as both $V_{T_{5}}$ and $V_{T_{1}}$ are already engaged in one relationship and are linked by the relationship between $V_{T_{9}}$ and $V_{T_{11}}$.

- $V_{T_{i^{\star}}}=V_{T_{1}}, V_{T_{j^{\star}}}=V_{T_{15}} \Rightarrow$ compatible.

Once variables ordered, the D-Vine model can be identified. It could then be used to perform MCS and to estimate different statistics of the transfer function.

\subsection{Summary of the proposed method}

Here are recalled the different steps of the proposed approach.

1. Computation of $N_{s}$ realizations of the random eigenvalue problem, from a random sample of size $N_{s}$ of the input parameters. Creation of the sample $\left(\lambda_{k}^{(r)}, \tilde{t}_{a c, k}^{(r)}, r \in\left[1, N_{s}\right], k \in\left[1, n_{k}\right]\right)$

2. Mode selection leading to a subset of modes of size $n_{i}$.

3. Determination of the variables ordering at the first level of the $D$-Vine decomposition.

4. Identification of the probability distribution of $\mathbf{V}_{T}$ from the sample $\left(\lambda_{k}^{(r)}, \tilde{t}_{a c, k}^{(r)}, r \in\left[1, N_{i}\right], k \in\left[1, n_{k}\right]\right)$. Let $\widehat{V}_{T}$ be the identified random vector. A large sample (size $N_{M C}=100,000$ ) is drawn from the probability distribution of $\widehat{V}_{T}$. This step is detailed in Fig. 2.

5. Corresponding realizations of the transfer function are calculated analytically, with Eq. (5).

6. Estimators of $\left|T_{a c}(\omega)\right|$ statistics are computed by MCS. In practice we focus on the $99 \%$ quantiles of $\left|T_{a c}\right|$.

The numerical cost of these different steps has to be discussed. From now on, all the presented results have been obtained with a quad core computer, with processors of $3.20 \mathrm{GHz}$ and memory of $16 \mathrm{~GB}$. As our objective is to deal with large finite element models, it is assumed that the resolution of a single realization of the random eigenvalue problem could last from few dozen of minutes to hours. Obviously, numerical cost of the first step, i.e. numerically solving $N_{s}$ eigenvalue problems, is linearly dependent on the cost of a resolution of a single realization of the random eigenvalue problem (acceleration methods such as the one proposed in [17] may be considered to improve the efficiency). Step 2 cost (forward modes selection) is approximately quadratic with respect to the number of candidate modes and linear with respect to the size of the identification sample $N_{s}$. In the worst numerical example we tested (number of candidates modes equal to 36 and $N_{s}=100$ ) it last 


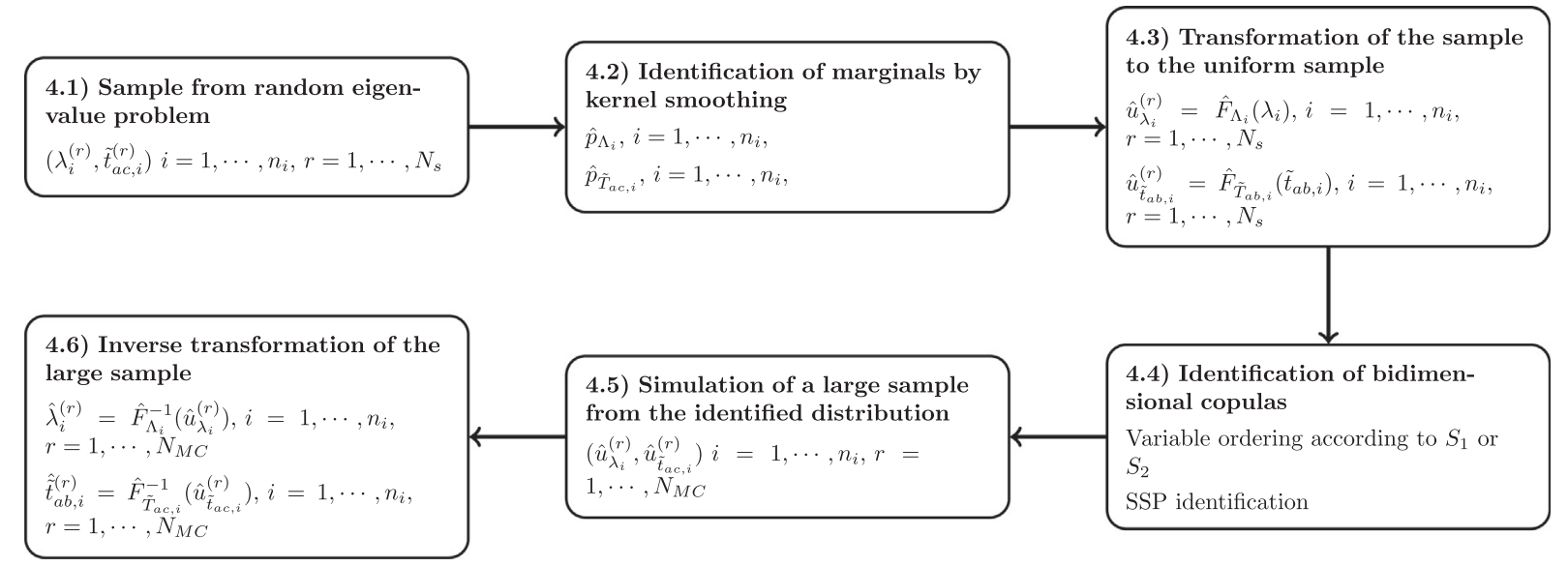

Fig. 2. Details of step 4: identification and simulation.

approximately for $13 \mathrm{~s}$, which is negligible. Cost of step 3 has to be considered only for strategy $S_{2}$. It appears that the greedy algorithm, used by strategy $S_{2}$, is very fast (in the following examples, the higher cost is less than one second for 15 selected modes and $N_{s}=100$ ) and this step cost is also negligible.

Numerical cost of step 4 mainly concerns the substeps (4.3) and (4.5). The total cost of this step seems linearly sensitive to the sample size $N_{s}$. Dependence to the number of selected modes $n_{i}$ is quadratic as the number of bidimensional copulas, involved in the decomposition, is equal to $n_{i}\left(n_{i}-1\right) / 2$. In the following examples, the shortest time for step 4 was approximately $18 \mathrm{~s}$ for $n_{i}=6$ and $N_{s}=10$, whereas the longest one is about $100 \mathrm{~s}$ for $n_{i}=11$ and $N_{s}=100$.

Numerical costs of steps 5 and 6 are mainly dependent on the sample size $N_{M C}$ and on the frequency discretization (step 5 is also slightly dependent of the number of selected modes but it is negligible for the range we study). In the following, we set $N_{M C}=100,000$ in order to obtain a correct approximation of the $99 \%$ quantiles of transfer function and frequency resolution is around 200 points. Let us add that these steps only involve computations of analytical transformations which justify such a large value for $N_{M C}$. It leads to approximately $60 \mathrm{~s}$ for steps 5 and 6.

Finally, in the worst case $\left(n_{i}=11\right.$ and $\left.N_{s}=100\right)$, the total cost of the approach is around 3 min. So, for large industrial structures, the cost of this approach remains negligible, compared to the one of a single random eigenvalue problem realization.

\section{Applications}

\subsection{Mass-spring-damper system}

\subsubsection{Description}

This section illustrates some points of the proposed methodology on a 20 DOF mass-spring-damper system (Fig. 3 presents it and gives notation).

Random masses are modeled by independent Gamma random variables of mean $1 \mathrm{~kg}$ and variation coefficient of $10 \%$. Random spring coefficients are modeled by independent Log-normal random variables of mean $1 \mathrm{~N} . \mathrm{m}^{-1}$ and variation coefficient of $10 \%$. Masses and spring coefficients are also independent. Finally, the probabilistic model of input parameters has 40 independent random variables. Moreover, it is added a modal damping, the shape of which is $c_{i}=2 \xi_{i} \sqrt{\Lambda_{i}}$ where $\xi_{i}=0.02$ for $i=1, \ldots, 20$.

The transmissibility between the DOF $u_{0}$ and $u_{1}$ is studied over the pulsation range $[0,1.3]$ rad.s ${ }^{-1}$. Fig. 4 presents the deterministic transmissibility modulus as well as the $99 \%$ quantiles computed by Monte Carlo Simulation with 100,000 simulations. These results are obtained using all the 20 modes and will be used as reference in the following.

Fig. 4 shows that, in this simple example, the modes are well separated and that there is almost no coupling phenomenon between them.

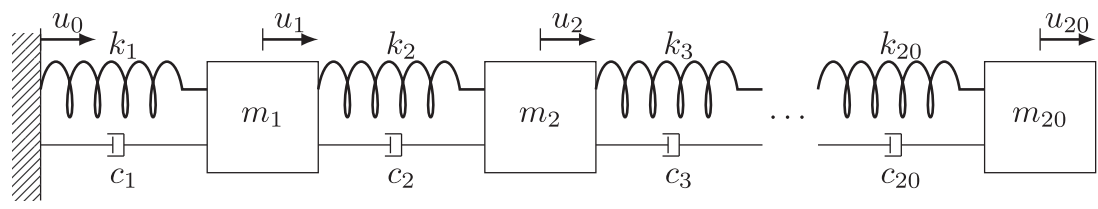

Fig. 3. 20 DOF mass-spring-damper system. 
A comparison between the proposed method and direct MCS is presented now. In order to quantify the accuracy of the different methods, the following criterion is used:

$$
\epsilon=\frac{1}{N_{p}} \sum_{k=1}^{N_{p}} \frac{\left|q_{99}^{r e f}\left(\omega_{k}\right)-\hat{q}_{99}\left(\omega_{k}\right)\right|}{q_{99}^{r e f}\left(\omega_{k}\right)}
$$

where $q_{99}^{\text {ref }}\left(\omega_{k}\right)$ is the reference $99 \%$ quantile at the pulsation $\omega_{k}$ and $\hat{q}_{99}$ its estimation by one of the methods. In practice $N_{p}=170$.

\subsubsection{Application of the identification approach}

The first step deals with the deterministic truncation. The upper boundary of the pulsation range of interest is $1.3 \mathrm{rad} . \mathrm{s}^{-1}$. Then are retained modes such as $\sqrt{\lambda_{k}}<1.3 \sqrt{2} \mathrm{rad}^{-1} \mathrm{~s}^{-1}$. In the deterministic case, this leads to select the 15 first modes. Hence, only those first 15 modes are computed for each realization of the random eigenvalue problem.

Concerning the modes selection step, by the forward methodology introduced in Section 4.1.2, Fig. 5(i) presents the values of $\hat{\epsilon}^{n_{i}} / \hat{\epsilon}^{0}$ for $n_{i}=0, \ldots, 15$, obtained with one sample of size $N_{s}$. Five values $N_{s}=10,30,50,70,100$ are tested, to quantify the sensitivity with respect to the sample size. To illustrate the differences between the selected modes, Fig. 5(ii) gives the number of differences between the selected subsets, for each subset size $n_{i}$. To do this, the reference family of selected subsets is obtained with a sample of size $N_{s}=100$, and the four other values of $N_{s}$ are compared with it. So, if for a given $N_{s_{0}}$, the difference is zero for a subset size $n_{i}$, it means that the $n_{i}$ selected modes are the same with sample $N_{s_{0}}$ and with sample of size $N_{s}=100$.

Fig. 5(i) shows that the accuracy increases continuously with respect to $n_{i}$. All the modes seem to influence the considered transfer function. It should be noted that this result was expected as, for such a simple example, all the modes are in the direction of excitation. Concerning influence of sample sizes $N_{s}$, Fig. 5(ii) reveals that subsets of size $n_{i}$ are almost composed of the same modes for all the sampling sizes (only one inversion appears for $N_{s}=10$ and $N_{s}=30$ when $n_{i}=13$ ). To conclude, the modes selection procedure is not relevant for this simple academic example and its interest will be highlighted in the next example. Based on this remark, the 15 first modes are kept to be part of the random vector $\mathbf{V}_{T}$, which is then of dimension 30.

Identification of the probability distribution of $\mathbf{V}_{T}$ by D-Vine decomposition is illustrated for the two strategies proposed in Section 4.2. The Kendall's $\tau$ correlation matrix is computed with the reference sample of size 100,000. Fig. 6(i) presents the results, where $L_{i}$ stands for variables $\Lambda_{i}$ and $T_{i}$ for variables $\widetilde{T}_{u_{1} u_{0}, i}$. Kendall's $\tau$ values are represented by circles which radius are proportional to correlation strength.

These results show that, even with independent random variables as in our model input, strong correlations appear between the modal parameters. In particular, all eigenvalues are positively correlated (yellow circles at each lines and columns of variables $\mathrm{L}_{i}$ ). One can also note that correlation between effective parameters is significant only for the first four modes. Another interesting point is that, here, effective parameters and eigenvalues are uncorrelated.

Identification strategies are now applied. To evaluate the dispersion of the different methods, 25 replicates are used. Table 1 presents the sum of absolute errors between the reference results and the Kendall's $\tau$ computed from the identified

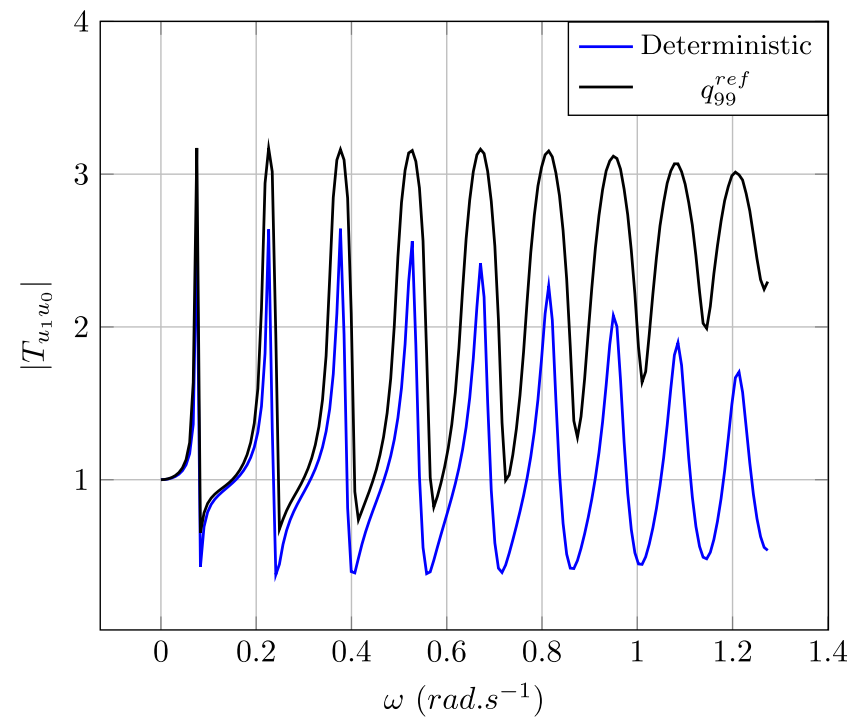

Fig. 4. $\left|T_{u_{1} u_{0}}\right|$ transmissibility modulus between the DOF $u_{0}$ and $u_{1}$. 


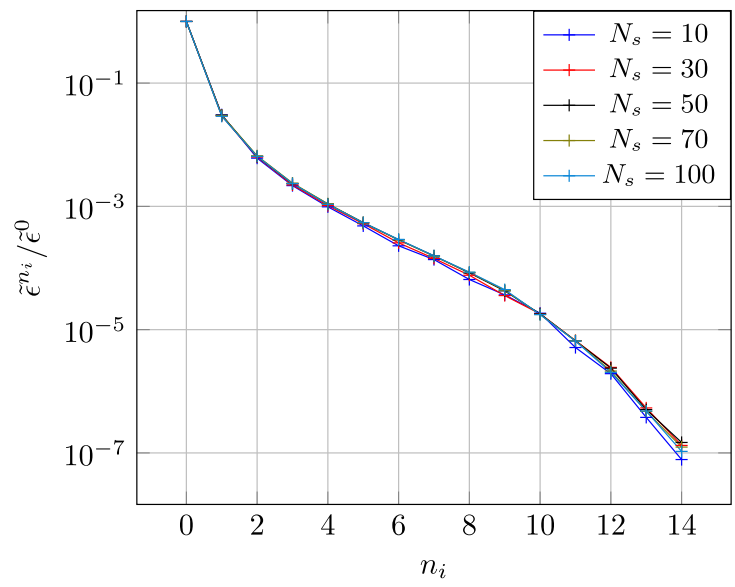

i)

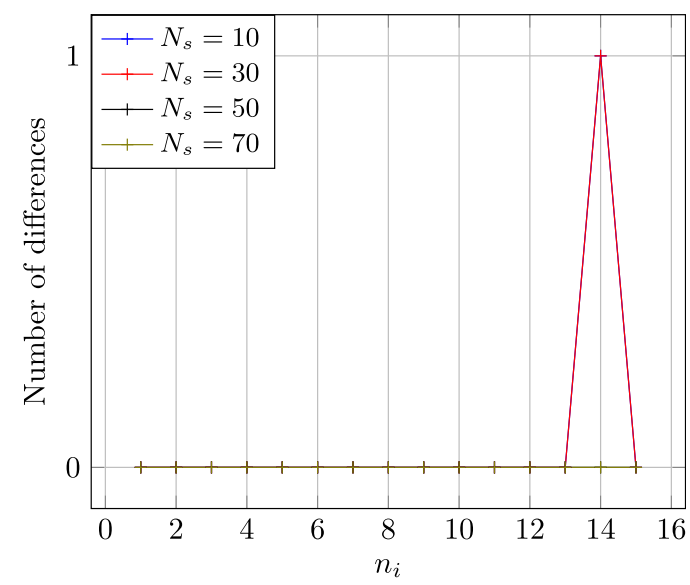

ii)

Fig. 5. (i) Evaluation of $\hat{\epsilon}^{n_{i}} / \hat{\epsilon}^{0}$ for $n_{i}=0, \ldots, 15$. (ii) Number of differences in selected subsets with respect to the sample size $N_{s}$.

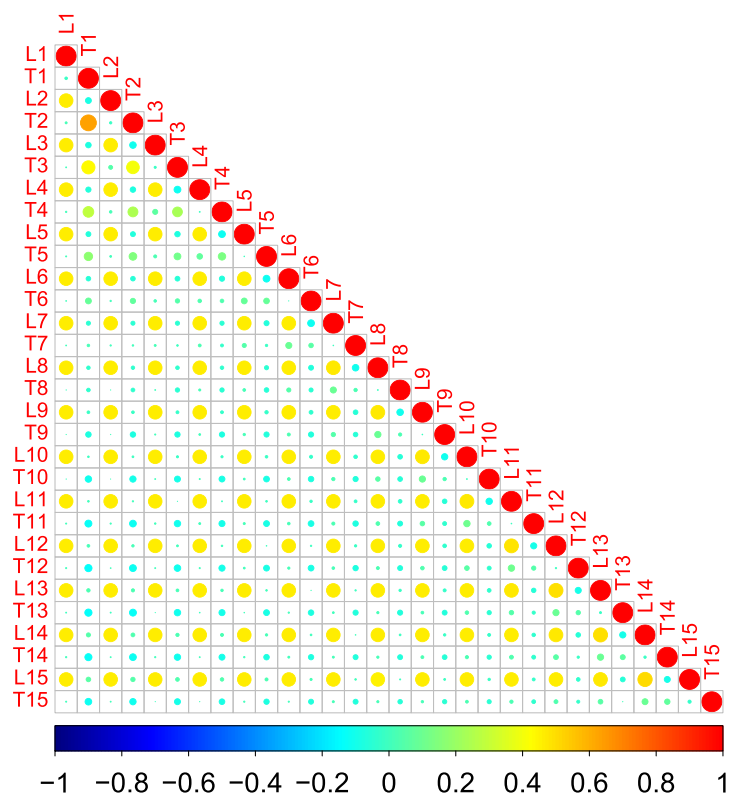

i)

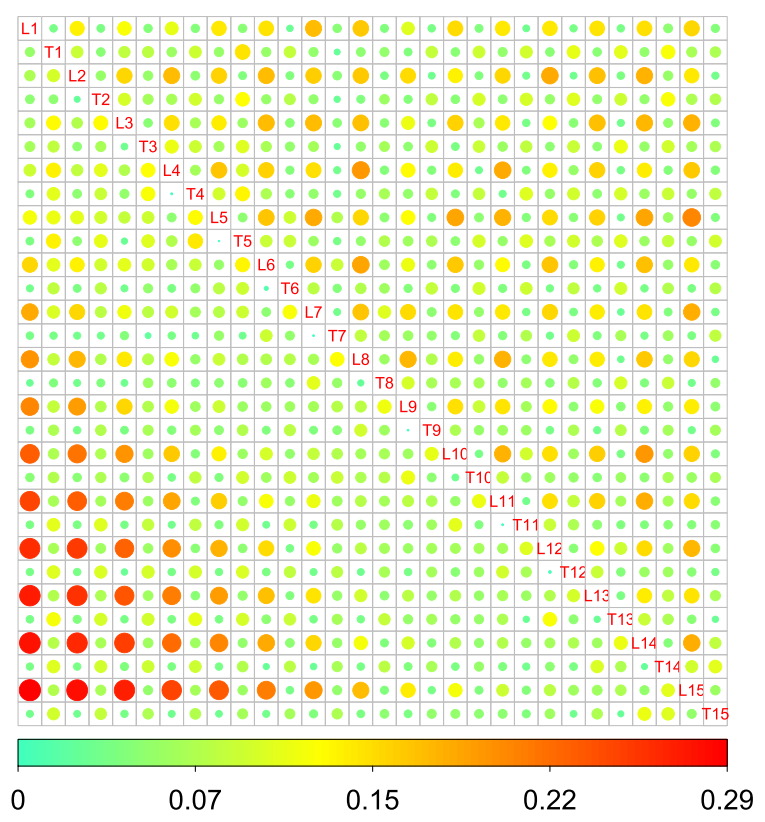

ii)

Fig. 6. (i) Reference Kendall's $\tau$ correlation matrix. (ii) Estimation of the mean absolute error in the computation of Kendall's $\tau$ by identification strategies $S_{1}$ (lower triangular part) and $S_{2}$ (upper triangular part) with $N_{s}=50$.

distribution for both strategies (evaluation of mean and its $95 \%$ confidence interval) with respect to the sample size $N_{s}$. Fig. 6 (ii) gives the mean absolute error between the reference results and the Kendall's $\tau$ computed from the identified distribution based on a samples of size $N_{s}=50$. Results of strategy $S_{1}$ are cast into the lower triangular part of the matrices, whereas results obtained by $S_{2}$ are cast into the upper triangular part of the matrices (the same samples are used for both strategies).

Table 1

Estimation of the mean of the relative errors sum on Kendall's $\tau$ for strategies $S_{1}$ and $S_{2}$.

\begin{tabular}{|c|c|c|c|}
\hline$N_{s}$ & 10 & 50 & 100 \\
\hline$S_{1}$ & $1.45 .10^{-1} \pm 6.35 .10^{-3}$ & $8.11 .10^{-2} \pm 3.73 .10^{-3}$ & $6.55 .10^{-2} \pm 2.74 .10^{-3}$ \\
\hline$S_{2}$ & $1.53 .10^{-1} \pm 9.80 .10^{-3}$ & $8.28 .10^{-2} \pm 3.48 .10^{-3}$ & $6.04 .10^{-2} \pm 1.97 .10^{-3}$ \\
\hline
\end{tabular}


Some conclusions can be drawn from these first results. Obviously, accuracy of the identification approach increases with the sample size for both strategies (as shown by Table 1). Moreover, it is difficult to identify which strategy performs better, but one can note that the two strategies do not focus on the same correlations and, consequently, the absolute error matrix is clearly not symmetric. More precisely, for strategy $S_{1}$, Fig. 6(ii) shows that identification errors are not homogeneous between pairs of variables. One can note that error increases with the distance to the first diagonal. This observation confirms that identification error increases with the level of the decomposition (we recall that for strategy $S_{1}$ variable ordering is the same as the one of Fig. 6(ii)). For the case of strategy $S_{2}$ absolute errors are spread almost homogeneously between correlated pairs of variables. With both strategies uncorrelated pairs of variables are perfectly identified.

We now discuss the influence of these differences in the computation of the 99\% quantiles of $\left|T_{u_{1} u_{0}}\right|$. Fig. 7 gives evolution of criterion defined by Eq. (11) for $N_{s}=10,30,50,70,100$. Results of both strategies are presented and a comparison with results obtained by direct Monte Carlo simulation with the same samples is provided. In order to measure the influence of dependence structure between modal parameters, Fig. 7 also presents results obtained by considering that all component of $\mathbf{V}_{T}$ are independent (caption Ind). Finally, dispersion of each methods is still evaluated by the use of 25 replicates (Fig. 7 presents estimation of mean values and their $95 \%$ confidence interval).

These results show that the 3 identification strategies outperform direct MCS. On this example, one can approximately stand that direct Monte Carlo method needs a sample two times larger than the one used by identification strategy to reach a comparable accuracy level. Comparison between the 3 identification strategies $\left(S_{1}, S_{2}\right.$ and $\left.I n d\right)$ points out that there is no relevant difference between them. This observation confirms that, in this example, the modes are well separated and that each one can be considered as an independent one DOF system with random parameters.

To conclude, the following observations can be made:

- Comparison between the identification strategies $S_{1}$ and $S_{2}$ illustrates the results of the literature about D-Vine identification and shows that variables ordering has a non negligible effect on the identification of the probability distribution of $\mathbf{V}_{T}$ (see Fig. 6(ii)).

- Interest of identification approaches compared to direct Monte Carlo simulation is shown in the context of small size identification samples. This results confirms the possible extension of the results about single DOF system presented in [23] and in [24].

- If modes are well separated, influence of correlation between modal parameters is negligible.

\subsection{Composite truss}

This section presents an industrial example, introduced in [46], in which it will be seen that the $99 \%$ quantile of the transfer function is influenced by the interaction between modes.

\subsubsection{Description}

The structure is a composite truss which model is presented Fig. 8(i).

Each tubular member of this reticular structure has an internal radius of $0.08 \mathrm{~m}$ and a thickness of $3 \mathrm{~mm}$. Longitudinal and transversal beams are $1 \mathrm{~m}$ long so the diagonal beams are $1.4142 \mathrm{~m}$ long. Each beam is discretized by 4 beam finite elements. Materials used is carbon/epoxy composite, the angle-ply lamination sequence is $\pm 45^{\circ}$. Beam axial Young's modulus $E$, shear stiffness $G$ and mass density $\rho$ are modeled by normal and exponentially correlated random fields, correlation dis-

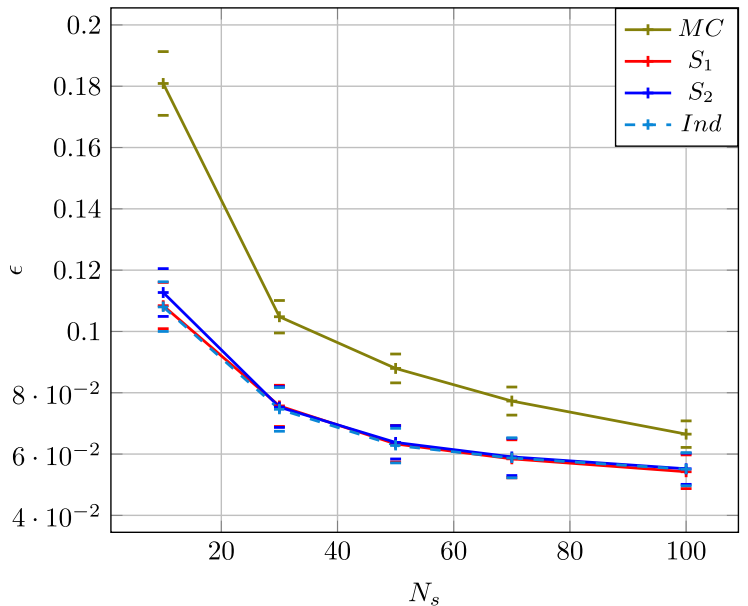

Fig. 7. Estimation of the mean of the criterion defined by Eq. (11) for the different methods. 


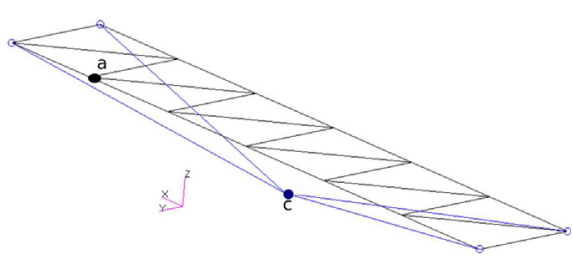

i)

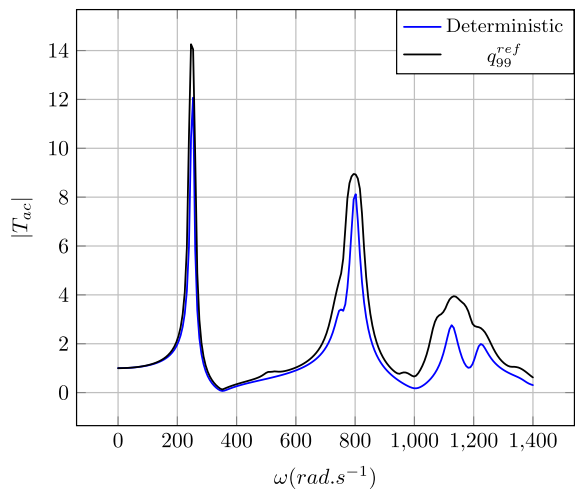

ii)

Fig. 8. (i) Geometric model of the truss. (ii) $\left|T_{a c}\right|$ transmissibility between the DOF $c$ and $a$ in the $Y$ direction.

tances are assumed equal to $0.25 \mathrm{~m}$ for the three random fields. Mean values and coefficients of variation of the three random fields are given in Table 2.

Discretization of the random fields is obtained by the midpoint method. This leads to a total of 300 random variables as input of the model. We assume modal damping with all damping coefficients equal to $\xi_{k}=0.02, k=1, \ldots, n_{i}$. Finally, the four corners of the structure are linked to the junction node $c$ with rigid elements.

We investigate the dynamic transmissibility between $Y$ translation DOF of node $c$ and $Y$ translation DOF of node $a$, over the pulsation range $] 0,1400] \mathrm{rad} . \mathrm{s}^{-1}$. The transfer function, obtained with the mean values of the input parameters, is presented Fig. 8(ii). In the deterministic case, 36 modes are used to compute this transfer function. Among these 36 modes, 21 modes have a natural pulsation greater than $1000 \mathrm{rad} . \mathrm{s}^{-1}$. This example is thus well designed to illustrate an application with a relatively important modal density. Fig. 8(ii) also presents the $99 \%$ quantile of $\left|T_{a c}\right|$ computed by direct MCS with a sample of size 100,000. These results will be used as reference in the following. Compared to the previous example, it is clear that modes are not so well separated, and that modes coupling phenomenon influences the $99 \%$ quantiles, especially for $\omega>1000 \mathrm{rad} . \mathrm{s}^{-1}$. Moreover, one can notice that two modes, which natural pulsations are approximatively $500 \mathrm{rad} . \mathrm{s}^{-1}$ and 980 rad. $\mathrm{s}^{-1}$, only appear on the $99 \%$ quantiles and not on the deterministic transfer function.

\subsubsection{Selection of relevant modes by forward selection}

The modes selection method developed in Section 4.1.2 is now applied. In order to quantify its sensitivity to the sample size, 5 sizes are tested $N_{s}=10,30,50,70,100$. Estimation of the criterion $\epsilon^{n_{i}}$ is given for $i=0, \ldots, 35$ (as $\epsilon^{n_{i}}=0$ for $n_{i}=n_{k}=36$ ). Fig. 9(i) displays the values of $\hat{\epsilon}^{n_{i}} / \hat{\epsilon}^{0}$ for $i=0, \ldots, 35$. These results confirm that only a small subset of modes is sufficient to get an accurate representation of a given random transfer function (an error of $10^{-6}$ is reach with about 10 modes). Another interesting point is that the main relevant modes are identified even with a low sample size. Additionally, there is almost no differences in the number of selected modes to reach an accuracy of $10^{-6}$ with respect to the sample size.

Concerning the differences in the selected modes for a given subset size $n_{i}$ with respect to $N_{s}$, as for the mass-springdamper example, Fig. 9(ii) gives the number of differences between the subset selected for $N_{s}=100$ and the 4 others sample sizes. Between $n_{i}=1$ and $n_{i}=19$, the differences number oscillates between 0 and 1 . Moreover, Fig. 9(i) shows that these 19 modes are precisely the most important ones. So, these results confirm the capability of the method to select the most relevant modes even with a sample of a rather small size.

In the following, 3 accuracy levels are tested for the construction of vector $\mathbf{V}_{T}$, say $\tilde{\epsilon}^{n_{i}} / \tilde{\epsilon}^{0}=10^{-4}, 10^{-5}, 10^{-6}$. Even if it appears that the subset composition is almost not dependent of the sample size for these accuracy levels, in the following, the same subset will be used for all sample sizes in order to observe convergence only with respect to $N_{s}$. Finally, we decide to retain subsets selected for $N_{s}=30$.

\section{Remark. Modes association and modes degenerative phenomenon.}

In the following modes association is performed by eigenvalues ascending order. Nevertheless, in order to allow a parametric identification of copulas, one has to identify mode crossing phenomenon. Let us illustrate this on this truss example.

Table 2

Definition of the characteristics of each random fields.

\begin{tabular}{ccc}
\hline Variable & Mean & $\mathrm{cv}(\%)$ \\
\hline$E$ & $8.13 \mathrm{GPa}$ & 10 \\
$G$ & $15.4 \mathrm{GPa}$ & 10 \\
$\rho$ & $1700 \mathrm{~kg} \cdot \mathrm{m}^{-3}$ & 5 \\
\hline
\end{tabular}




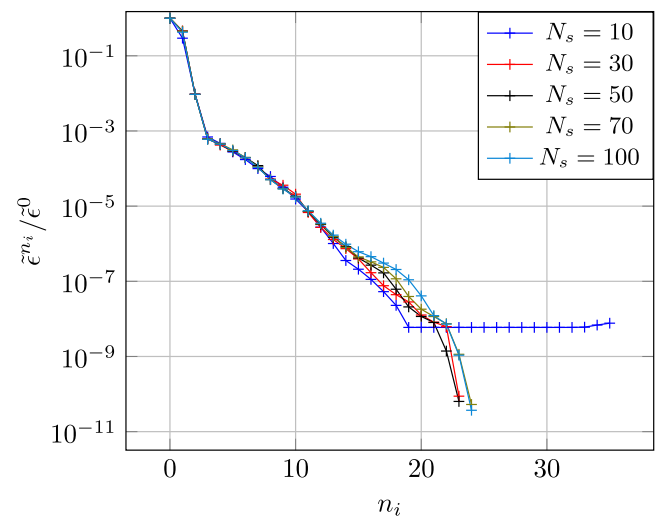

i)

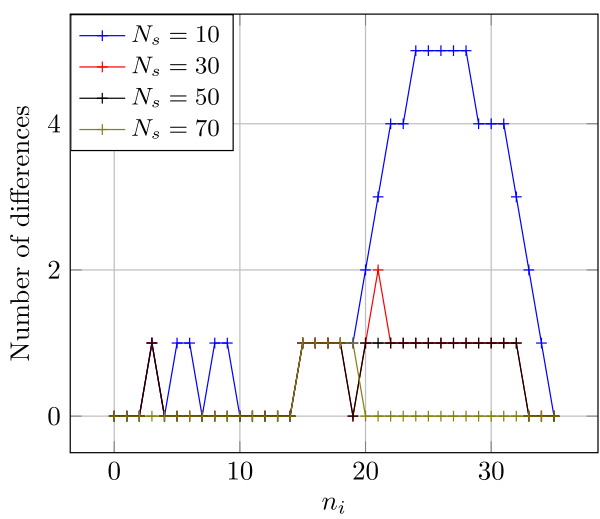

ii)

Fig. 9. (i) Evaluation of $\tilde{\epsilon}^{n_{i}} / \tilde{\epsilon}^{0}$ for $n_{i}=0, \ldots, 35$. (ii) Number of differences in selected subsets with respect to the sample size $N_{s}$.

The two first selected modes are the second and the third of the structure. The dependence graph of their effective parameters is presented Fig. 10 for $N_{s}=30$. This graph is typical of a mode crossing phenomenon with an inversion between 2 close modes. One can see that the effective parameter of mode 2 approximately belongs to $[0.5 ; 0.6]$ when the effective parameter of mode 3 is zero, and that the effective parameter of mode 3 approximately belongs to $[0.5 ; 0.6]$ when the effective parameter of mode 2 is zero. It is clear that these realizations come from one mode with effective parameter equal to zero and one mode with effective parameter in $[0.5 ; 0.6]$. In this case, the "two" modes are concatenated into one by only considering the non zero realizations of the effective parameters (one can check that the sum of these non zero realizations is equal to $N_{s}$ ).

Table 3 gives the subsets sizes for each level of accuracy, after elimination of the fake modes due to mode crossing.

\subsubsection{Variable ordering and identification of the probability distribution of $\mathbf{V}_{T}$}

For sake of clarity, we propose to set $\hat{\epsilon}^{n_{i}} / \hat{\epsilon}^{0}=10^{-4}$ which leads to the selection of 6 modes. This accuracy level is retained in order to discuss the identification process with a limited number of variables. The results we obtained with the other accuracy levels, are discussed in Section 5.2.5. Mean eigenvalues of these 6 modes, estimated by direct MCS with 100,000

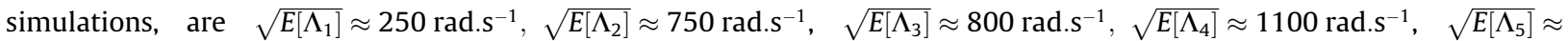
1120 rad.s ${ }^{-1}$ and $\sqrt{E\left[\Lambda_{6}\right]} \approx 1150$ rad.s ${ }^{-1}$.

The reference Kendall's $\tau$ matrix, estimated by MCS with 100,000 simulations, is presented Fig. 11. The lower triangular part gives the absolute values of Kendall's $\tau$ whereas the upper presents dependence graphs from a subsample of size 100 (only 100 points were used to allow a correct visibility of points density).

This figure shows that only a small number of pairs of variables are correlated. Indeed only 17 pairs are such that $\left|\tau_{i j}\right| \geqslant 0.3$. A study of the dependence structures gives relevant informations on modes coupling phenomenon. For example, eigenvalues $\Lambda_{4}$ and $\Lambda_{5}\left(L_{4}\right.$ and $L_{5}$ in Fig. 11) are positively correlated, which implies that the peaks, produced by their dynamic amplification factor on the transfer function, have a tendency to move "together". The probability of large increase of the transfer function due to a coupling is thus limited. Moreover, their effective parameters $\left(T_{4}\right.$ and $T_{5}$ in Fig. 11) are negatively correlated. Once again, this dependence structure is going to limit the probability of large increase of the transfer function. This effect is also very important between modes 2 and $3\left(T_{2}\right.$ and $T_{3}$ and in Fig. 11). It is also interesting to observe

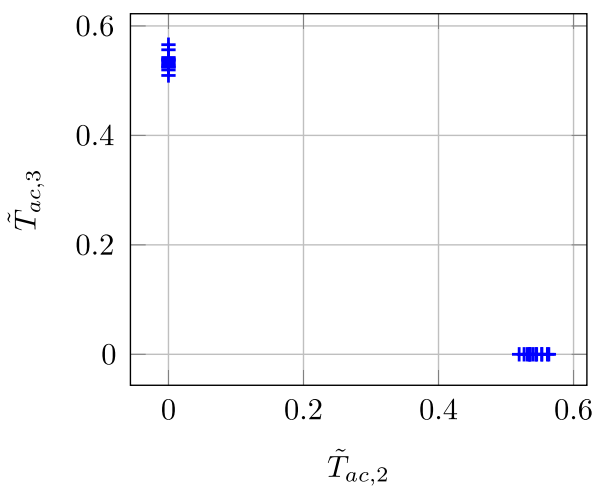

Fig. 10. Illustration of mode crossing. Dependence graph of $\widetilde{T}_{a c, 2}$ and $\widetilde{T}_{a c, 3}$, with $N_{s}=30$. 
Table 3

Number of modes in the selected subsets in function of the targeted accuracy $\tilde{\epsilon}^{n_{i}} / \tilde{\epsilon}^{0}$ computed with $N_{s}=30$.

\begin{tabular}{cc}
\hline$\tilde{\epsilon}^{n_{i}} / \tilde{\epsilon}^{0}$ & $n_{i}$ \\
\hline $10^{-4}$ & 6 \\
$10^{-5}$ & 9 \\
$10^{-6}$ & 11 \\
\hline
\end{tabular}

\begin{tabular}{|c|c|c|c|c|c|c|c|c|c|c|c|}
\hline L1 & 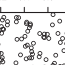 & & & $\because 800$ & & & & & 8 & & \\
\hline 0.10 & T1 & 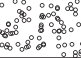 & 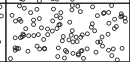 & of & & (6) & & $\begin{array}{l}80880 \\
\end{array}$ & 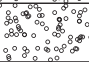 & 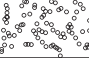 & \\
\hline 0.43 & $\ldots$ & L2 & 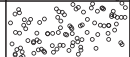 & 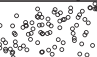 & & 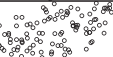 & 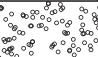 & 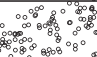 & 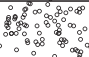 & 0 & \\
\hline & & 0.25 & T2 & 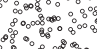 & & 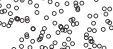 & & & & & \\
\hline 0.48 & 0.10 & 0.34 & & L3 & & & & & 60 & & \\
\hline - & 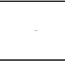 & 0.17 & 0.71 & sor & T3 & 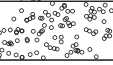 & & & & & \\
\hline 0.36 & & 0.31 & 0.14 & 0.30 & 0.21 & L4 & & & & & \\
\hline & ous & oon & - & & & ... & T4 & & & & \\
\hline 0.52 & 0.12 & 0.30 & 0.20 & 0.43 & 0.22 & 0.53 & on & L5 & - & 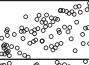 & \\
\hline & 0.16 & 0.20 & 0.12 & & & $\cdots$ & 0.51 & 0.15 & T5 & & \\
\hline 0.41 & 0.15 & 0.18 & 0.12 & 0.31 & 0.16 & 0.30 & 0.14 & 0.46 & - & L6 & \\
\hline - & 0.14 & - & $\cdots$ & & $\ldots$ & $\ldots$ & 0.17 & & 0.17 & 0.30 & Tt \\
\hline
\end{tabular}

Fig. 11. Reference Kendall's $\tau$ matrix.

the various shapes of the dependence structures. For example, dependence structure between $\Lambda_{1}$ and $\Lambda_{3}\left(L_{1}\right.$ and $L_{3}$ in Fig. 11) presents strong correlation for higher values whereas small values are almost not correlated. Parametric bivariate copulas are thus a relevant tool to describe those dependence structures.

After these qualitative remarks, we compare the results in the identification of the probability distribution of $\mathbf{V}_{T}$ by strategies $S_{1}$ and $S_{2}$. As for the previous example, Fig. 12 presents the mean absolute error between the reference results and the Kendall's $\tau$, computed from the identified distribution based on a sample of size $N_{s}=100$ (means are evaluated with 25 replicates). Results of strategy $S_{1}$ are cast into the lower triangular part of the matrices whereas $S_{2}$ ones are cast into the upper triangular part.

Table 4 gives the estimation of the sum of the absolute errors in the computation of Kendall's $\tau$, for both strategies (mean values and their $95 \%$ confidence intervals estimated over the 25 replicates).

The remarks done on the first example are confirmed by the results of Fig. 12 and Table 4. First of all, Fig. 12 shows that error matrix obtained with $N_{s}=100$ is not symmetric. Moreover, one can note that conclusion drawn with the first example about homogeneity of the error level obtained with strategy $S_{2}$ is confirmed. Table 4 shows that both strategies give very close results. But, for small sample sizes, dispersion of the first strategy is smaller than the one of $S_{2}$. In contrary, for $N_{s}=100, S_{2}$ performs better and is less dispersed.

Some results about strategy $S_{2}$, obtained with a single sample, are now detailed. In particular, application of algorithm described in Section 4.2 leads to the following order:

- $N_{s}=10, \Lambda_{3}-\widetilde{T}_{a c, 1}-\Lambda_{1}-\widetilde{T}_{a c, 5}-\widetilde{T}_{a c, 4}-\Lambda_{6}-\Lambda_{5}-\widetilde{T}_{a c, 3}-\widetilde{T}_{a c, 2}-\Lambda_{2}-\widetilde{T}_{a c, 6}-\Lambda_{4}$

- $N_{s}=50, \Lambda_{4}-\Lambda_{5}-\Lambda_{6}-\Lambda_{1}-\Lambda_{3}-\Lambda_{2}-\widetilde{T}_{a c, 2}-\widetilde{T}_{a c, 3}-\widetilde{T}_{a c, 6}-\widetilde{T}_{a c, 1}-\widetilde{T}_{a c, 5}-\widetilde{T}_{a c, 4}$

- $N_{s}=100, \widetilde{T}_{a c, 1}-\widetilde{T}_{a c, 5}-\widetilde{T}_{a c, 4}-\widetilde{T}_{a c, 6}-\Lambda_{6}-\Lambda_{4}-\Lambda_{5}-\Lambda_{1}-\Lambda_{3}-\Lambda_{2}-\widetilde{T}_{a c, 2}-\widetilde{T}_{a c, 3}$

It is notable that, according to the reference results, on these 11 bivariate relationships, 3 are such that $\left|\tau_{i j}\right| \geqslant 0.3$ for $N_{s}=10,7$ for $N_{s}=50$ and 8 for $N_{s}=100$. This clearly indicates that a sample of size $N_{s}=10$ is here not sufficient to detect the correlated pairs of variables. The results obtained with $N_{s}=50$ and $N_{s}=100$ reveal the capability of strategy $S_{2}$ to find a "path" that optimizes the correlation between pairs of variables. Additionally, with $N_{s}=100$, upon the $2 n_{i}\left(2 n_{i}-1\right) / 2=66$ 


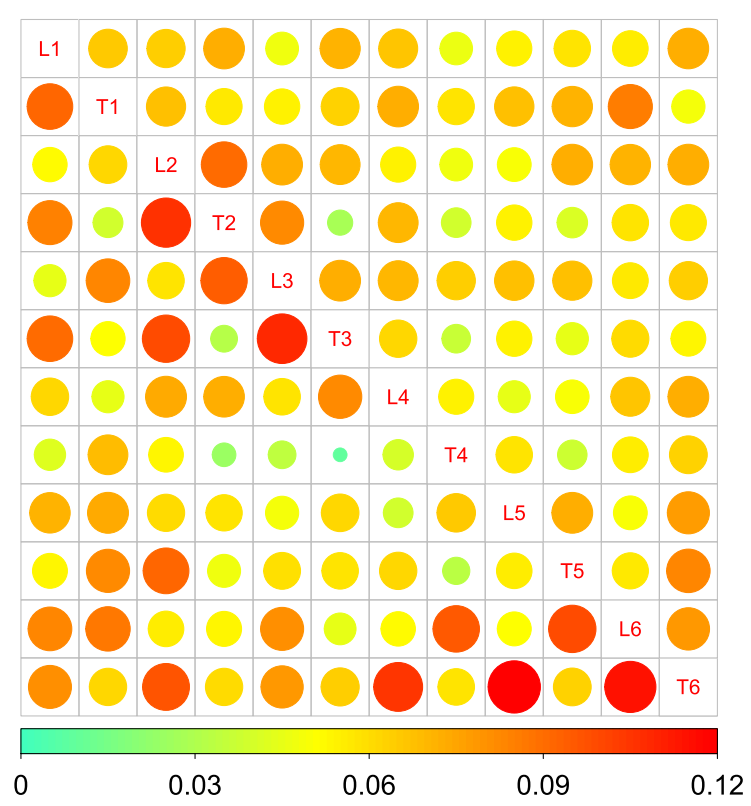

Fig. 12. Absolute error in the computation of Kendall's $\tau$ by identification strategies $S_{1}$ (lower triangular part) and $S_{2}$ (upper triangular part) with $N_{s}=100$.

Table 4

Estimation of the mean of the relative errors sum on Kendall's $\tau$ for strategies $S_{1}$ and $S_{2}$.

\begin{tabular}{cccc}
\hline$N_{S}$ & 10 & 50 & 100 \\
\hline$S_{1}$ & $1.60 .10^{-1} \pm 7.44 .10^{-3}$ & $8.86 .10^{-2} \pm 5.36 .10^{-3}$ & $6.55 .10^{-2} \pm 4.24 .10^{-3}$ \\
$S_{2}$ & $1.62 .10^{-1} \pm 1.23 .10^{-2}$ & $9.07 .10^{-2} \pm 5.84 .10^{-3}$ & $6.04 .10^{-2} \pm 4.01 .10^{-3}$ \\
\hline
\end{tabular}

bivariate copulas that must be identified, 36 are independent ones. The 30 others are divided in 12 gaussian copulas, 7 Gumbel copulas, 5 Clayton copulas, 4 Frank copulas, 1 Student copula and 1 Joe Copula. This illustrates the important variety of dependence structures involved in a random eigenvalues problem. Moreover, at the first level of the decomposition, none of the bivariate copulas is an independent one, which confirms again the capability of strategy $S_{2}$.

\subsubsection{Estimation of the $99 \%$ quantiles of the transfer function}

Estimation of the $99 \%$ quantiles of $\left|T_{a c}\right|$ by the two identification strategies is now studied. These strategies are compared with a direct Monte Carlo method. We precise that the same 6 modes are used in Monte Carlo method. It means only the simulation methods are compared, and not the error due to the modes selection procedure. As previously, the methods variability is quantified by the use of 25 replicates. Results are presented Fig. 13.

The first obvious remark is that an identification approach that would assume independent components for random vector $\mathbf{V}_{T}$ is not relevant. This shows that, when modes are not separated, dependence structure between modal parameters strongly influence the behavior of the random transfer function. Results obtained with strategies $S_{1}$ and $S_{2}$ confirm this interpretation. Moreover, as for the previous example, strategies $S_{1}$ and $S_{2}$ outperform direct Monte Carlo method even if the benefit of identification methods decreases as the sample size increases.

The above comparison criterion is very global with respect to pulsation $\omega$. Now, a local comparison is provided by computing, with each identification strategy, the $99 \%$ quantiles of $\left|T_{a c}\right|$ from the same samples of size $N_{s}=30$, see Fig. 14 (mean over the 25 replicates). We recall that, on this figure, the reference results are obtained by direct MCS with a sample of size 100,000 .

First of all, for $\omega<1000 \mathrm{rad} . \mathrm{s}^{-1}$, the 3 identification approaches give very close and accurate results (one can also note the effect of mode selection around $\omega=500 \mathrm{rad} . \mathrm{s}^{-1}$ ). But, for $\omega>1000 \mathrm{rad} . \mathrm{s}^{-1}$, the number of considered modes is more important and the coupling between them not negligible. So, assuming independent components for the random vector $\mathbf{V}_{T}$ leads to an overestimation of the $99 \%$ quantiles. In contrary, strategies $S_{1}$ and $S_{2}$ give more relevant results. Nevertheless, compared to the reference result, it can be noted that both strategies slightly overestimate the $99 \%$ quantiles around 1100 rad. ${ }^{-1}$. To improve these results, the next section investigates the effect of increasing the number of considered modes.

\subsubsection{Increasing the number of modes}

This part only focuses on the estimation of the $99 \%$ quantiles of the transfer function. Fig. 15 presents the estimation of criterion defined by Eq. (11) (as usually, estimation is performed over 25 replicates), for the 2 other accuracy levels (see 


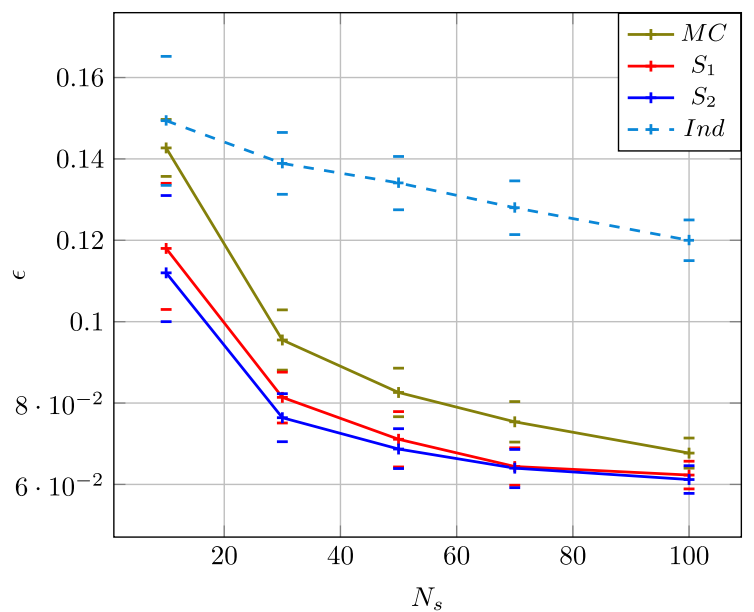

Fig. 13. Estimation of the mean of the criterion defined by Eq. (11) for the different methods $n_{i}=6$.

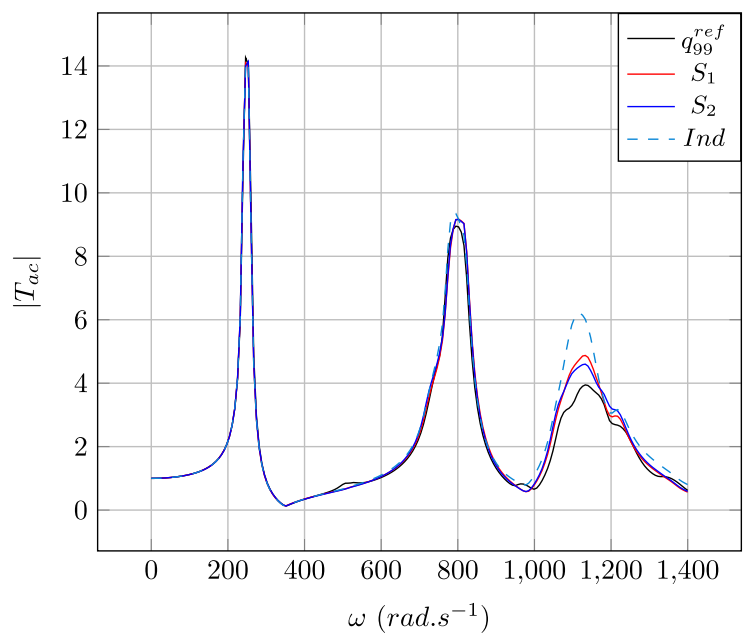

Fig. 14. Comparison of the estimators of the $99 \%$ quantiles of $\left|T_{a c}\right|$ computed by different methods. Mean values estimated on 25 replicates with $N_{s}=30$.

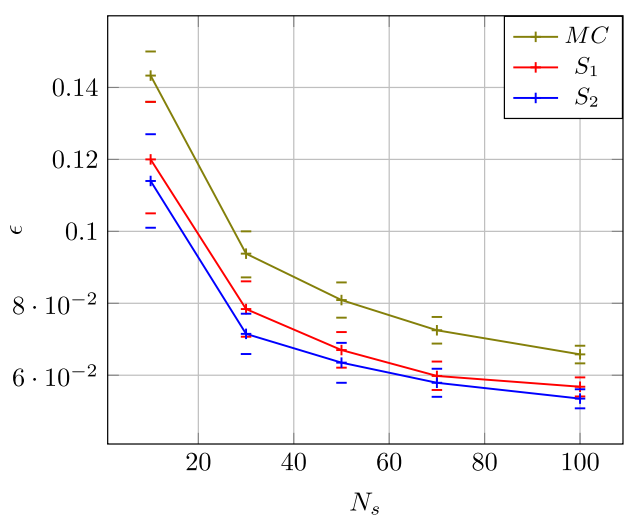

i)

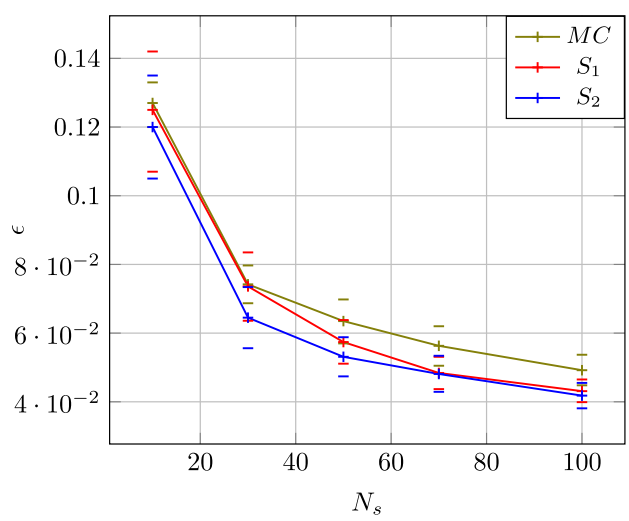

ii)

Fig. 15. Estimation of the mean of the criterion defined by Eq. (11) for the different methods. (i) $n_{i}=9$, (ii) $n_{i}=11$. 


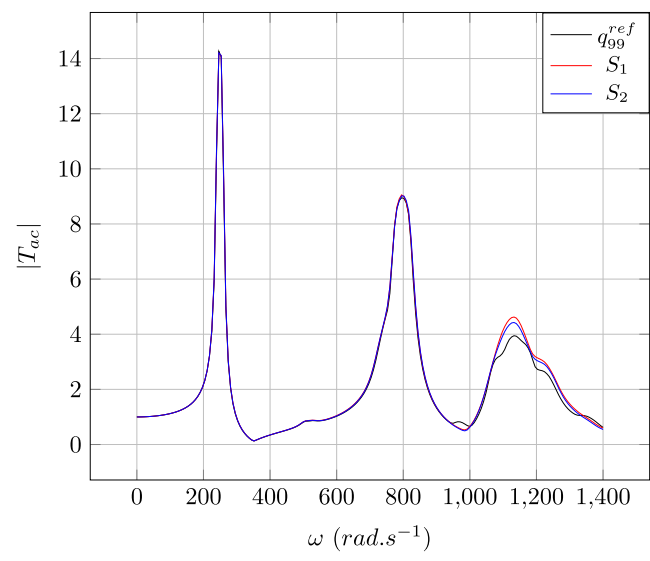

i)

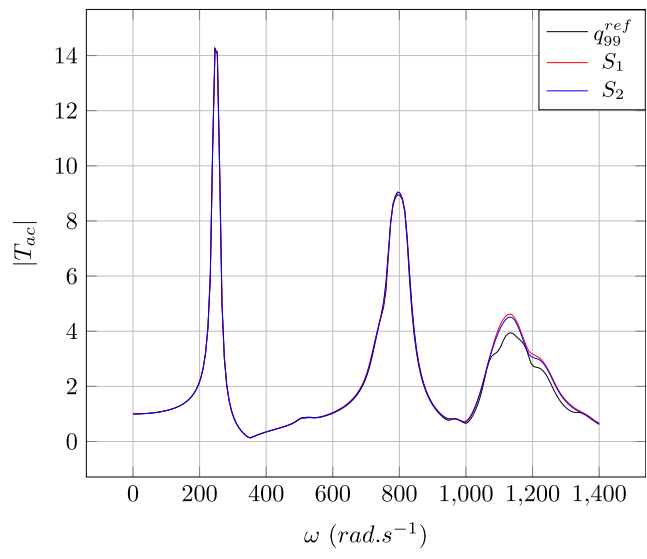

ii)

Fig. 16. Comparison of the estimators of the $99 \%$ quantiles of $\left|T_{a c}\right|$ computed by strategies $S_{1}$ and $S_{2}$. Mean values estimated on 25 replicates with $N_{s}=30$. (i) $n_{i}=9$, (ii) $n_{i}=11$.

Table 3) leading to $n_{i}=9,11$. As for the last example, comparison with direct MCS is performed with the same number of modes.

One can note that accuracy of the results obtained by direct MCS and by strategies $S_{1}$ and $S_{2}$ increases with the number of considered modes. Nevertheless, even if the proposed approaches perform better than direct MCS, it seems that the improvement is more important in the case of the MCS, especially from $n_{i}=9$ to $n_{i}=11$.

Finally, Fig. 16 presents a comparison of the estimators of the 99\% quantiles of $\left|T_{a c}\right|$ computed by strategies $S_{1}$ and $S_{2}$ with $N_{s}=30$ and $n_{i}=9$ (Fig. 16(i)) and $n_{i}=11$ (Fig. 16(ii)). Once again, the reference results are obtained by direct MCS with a sample of size 100,000 . It is notable that increasing the number of modes from $n_{i}=6$ (Fig. 14 ) to $n_{i}=9$ (Fig. $16(\mathrm{i})$ ) allows, first, to catch the mode around 500 rad. $\mathrm{s}^{-1}$ and second, to increase the accuracy for $\omega>1000$ rad. $\mathrm{s}^{-1}$ compared to the results presented by Fig. 14. Moreover, increasing the number of modes to $n_{i}=11$ (Fig. 16(ii)), only improves the results around 950 rad. $\mathrm{s}^{-1}$ but does not improve it for $\omega>1000$ rad.s $^{-1}$. This observation confirms the results presented in Fig. 15 . One could also note that, as for the other numerical examples, strategy $S_{2}$ performed slightly better that $S_{1}$.

This final results illustrate a limit of the proposed approach. Indeed, increasing the number of considered modes from $n_{i}=9$ to $n_{i}=11$ does not improve the results for $\omega>1000 \mathrm{rad}^{-1} \mathrm{~s}^{-1}$. This can be explained by the fact that the transfer function for these pulsations is influenced by too many random modes and that the proposed strategy failed to properly identify such a large probability distribution (dimension 22). Some improvements are proposed in conclusion to tackle this issue.

\section{Conclusion}

The work presented in this article focuses on two objectives. First one is the identification of the probability distribution of the modal parameters (eigenvalues and effective parameters) of a given random transfer function. To reach this goal a method to construct and identify a D-Vine decomposition of this probability distribution is proposed. Numerical examples illustrate the various dependence structures involved in the probability distribution of the modal parameters and the capabilities of the proposed approach in their identification. Hence, the adaptation of the D-Vine construction presented in this article is a relevant tool for identification of modal parameters probability distribution. Second objective is the construction of estimator of the $99 \%$ quantiles of the random transfer function by post processing of the identified modal parameters probability distribution. According to the numerical examples presented in Section 5 the proposed estimator outperforms the classical Monte Carlo estimators in all the studied cases. Theoretical developments of this result is a perspective of this work and it could be noted that a first attempt is proposed, for one dimensional case, in [24]. Nevertheless, it seems that the benefit of the approach decreases with the number of modes i.e. the size of the identification problem. To this respect one can conclude that the proposed approach is well suited to deal with vibration study of large industrial structures under parametric uncertainty involving a large number of random variables but a limited number of modes. Moreover, we would like to emphasize that, in its actual form, the proposed identification procedure is only suited to deal with a fixed design of the structure under study. In order to consider design under uncertainty issue, the identification procedure has to be done for all new tested designs, which is not efficient. However, a relevant way to achieve the identification of the probability distribution of modal parameters over a design space could be to consider the use of spacial Vine (see [47]). This enhancement for structural dynamics must be seen as a perspective of this work.

Finally, two extensions of this work are proposed. First one concerns the adaptation to a larger number of modes. Indeed, even if the modes selection step presented in Section 4.1.2 aims at reducing the number of relevant modes for the identification, in some cases, this reduction may not be sufficient to allow a correct identification of the probability distribution (see 
Section 5.2.5). With respect to this issue, an approach has been proposed in [23], which aims at splitting the identification problem into several identification problems of smaller size by selecting only the most critical modes interaction. Second one, is the evaluation of the dispersion of the proposed estimators. Indeed, in the present work, replicates are used to characterize dispersion which is not relevant for practical applications. In $[23,24]$ construction of bootstrap confidence interval is presented to characterize the dispersion of the $99 \%$ quantile estimators at a reasonable computational cost.

\section{Acknowledgment}

This work was supported by Centre National d'Etudes Spatiales and Thales Alenia Space. The authors would like to thank A. Giovannini, J. Buffe and F. Buffe, for fruitful discussions.

\section{References}

[1] B. Sudret, Uncertainty Propagation and Sensitivity Analysis in Mechanical Models - Contributions to Structural Reliability and Stochastic Spectral Methods, Habilitation á diriger des recherches, Université Blaise Pascal, Clermont-Ferrand, France, 2007.

[2] M. Capitaine, Matrices aléatoires, Lecture Notes, 2012.

[3] G. Anderson, A. Guionnet, O. Zeitouni, An Introduction to Random Matrices, Cambridge University Press, 2011.

[4] S. Adhikary, L. Pastur, A. Lytova, J. Du Bois, Eigenvalue density of linear stochastic dynamical systems: a random matrix approach, J. Sound Vib. 331 (2012) 1042-1058.

[5] C. Soize, A nonparametric model of random uncertainties for reduced matrix models in structural dynamics, Probab. Eng. Mech. 15 (3) (2000) 277-294.

[6] C. Soize, Random matrix theory for modeling uncertainties in computational mechanics, Comput. Methods Appl. Mech. Eng. 194 (12-16) (2005) 13331366.

[7] M. Shinozuga, C.J. Astill, Random eigenvalue problems in structural analysis, AIAA J. 10 (4) (1972) $456-462$.

[8] R.G. Ghanem, P.D. Spanos, Stochastic Finite Elements: A Spectral Approach. Civil, Mechanical and Other Engineering Series, Dover Publications, 2003.

[9] C. Soize, R. Ghanem, Physical systems with random uncertainties: chaos representations with arbitrary probability measure, SIAM J. Sci. Comput. 26 (2) (2004) 395-410.

[10] R. Ghanem, D. Ghost, Eigenvalue analysis of a random frame, in: Structural Dynamics: EURODYN 2002, 2002, pp. $341-346$.

[11] S. Adhikari, Joint statistics of natural frequencies of stochastic dynamic systems, Comput. Mech. 40 (4) (2007) 739-752.

[12] G. Georgiou, A. Manan, J.E. Cooper, Modeling composite wing aeroelastic behavior with uncertain damage severity and material properties, Mech. Syst. Signal Process. 32 (2012) 32-43 (Uncertainties in Structural Dynamics).

[13] H. Xu, S. Rahman, Decomposition method for structural reliability analysis, Probab. Eng. Mech. 20 (2005) 239-250.

[14] S. Rahman, A solution of the random eigenvalue problem by a dimensional decomposition method, Int. J. Numer. Methods Eng. 67 (2006) 1318-1340.

[15] G.I. Schuëller, L. Pichler, On the treatment of finite element structures in stochastic linear dynamics using a mode-based meta-model, Eng. Struct. 33 (2) (2011) 391-400

[16] M. Chevreuil, A. Nouy, Model order reduction based on proper generalized decomposition for the propagation of uncertainties in structural dynamics, Int. J. Numer. Methods Eng. 89 (2012) 241-268.

[17] G.S. Székely, G.I. Schuëller, Computational procedure for a fast calculation of eigenvectors and eigenvalues of structures with random properties, Comput. Methods Appl. Mech. Eng. 191 (2001) 799-816.

[18] G.S. Szekely, H.J. Pradlwarter, G.I. Schuëller, Random eigenvalue problems for large systems, Comput. Struct. 80 (2002) $2415-2424$.

[19] T. Bedford, R.M. Cooke, Probability density decomposition for conditionally dependent random variables modeled by vines, Ann. Math. Artif. Intell. 32 (2001) 245-268.

[20] T. Bedford, R.M. Cooke, Vines: a new graphical model for dependent random variables, Ann. Stat. 30 (2002) 1031-1068.

[21] R. Nelsen, An Introduction to Copulas, Springer, 2006.

[22] A. Girard, N. Roy, Structural Dynamics in Industry, Wiley-ISTE, 2007.

[23] S. Dubreuil, M. Salaün, E. Rodriguez, F. Petitjean, Construction of frequency response function confidence interval by a semi analytical approach, in: Uncertainty in Structural Dynamics. ISMA-USD, 2014, pp. 4637-4652.

[24] S. Dubreuil, Superposition modale probabiliste: application au dimensionnement des structures spatiales (Ph.D. Thesis), Universit'e de Toulouse, 2014.

[25] D. Afolabi, Modal Interaction in Linear Dynamic Systems Near Degenerate Modes. Technical Report, Purdue University Indianapolis, 1991.

[26] Jonathan Luke du Bois, Sondipon Adhikari, Nick A.J. Lieven, Eigenvalue curve veering in stressed structures: an experimental study, J. Sound Vib. 322 (4-5) (2009) 1117-1124.

[27] A.W. Leissa, On a curve veering aberration, J. Appl. Math. Phys. 25 (1974) 99-111.

[28] N.C. Perkins, C.D. Mote, Comments on a curve veering in eigenvalue problems, J. Sound Vib. 106 (3) (1986) $451-463$.

[29] E. Balmes, High modal density, curve veering, localization: a different perspective on the structural response, J. Sound Vib. 161 (2) (1993) 358-363.

[30] A. Gallina, L. Pichler, T. Uhl, Enhancement meta-modelling technique for analysis of mode crossing, mode veering and mode coalescence in structural dynamics, Mech. Syst. Signal Process. 25 (7) (2011) 2297-2312.

[31] D. Bettebghor, F-H Leroy, Overlapping radial basis function interpolants for spectrally accurate approximation of functions of eigenvalues with application to buckling of composite plates, Comput. Math. Appl. 67 (10) (2014) 1816-1836.

[32] H. Joe, Multivariate Models and Dependence Concepts, Chapman \& Hall, London, 1997.

[33] R. Lebrun, A. Dutfoy, A generalization of the Nataf transformation to distributions with elliptical copula, Probab. Eng. Mech. 24 (2009) 172-178.

[34] R. Lebrun, A. Dutfoy, Do Rosenblatt and Nataf isoprobabilistic transformation really differ?, Probab Eng. Mech. 24 (2009) 577-584.

[35] R. Lebrun, A. Dutfoy, An innovating analysis of the Nataf transformation from the copula viewpoint, Probab. Eng. Mech. 24 (2009) 312-320.

[36] A. Sklar, Fonctions de répartition à $n$ dimensions et leurs marges, Publ. l'Inst. Stat. l'Univ. Paris 8 (1959) $229-231$.

[37] J. MacKay, C. Genest, The joy of copulas: bivariate distributions with uniform marginals, Am. Stat. 40 (1986) $280-283$.

[38] K. Aas, C. Czado, A. Frigessi, H. Bakken, Pair-copula constructions of multiple dependence, Insur.: Math. Econ. 44 (2009) $182-198$.

[39] I. Hobæk Haff, K. Aas, A. Frigesi, On the simplified pair-copula construction - simply useful or too simplistic?, J Multivar. Anal. 101 (2010) 1296-1310.

[40] J. Stöber, H. Joe, C. Czado, Simplified pair copula constructions - limitations and extensions, J. Multivar. Anal. 119 (2013) $101-118$.

[41] H. Akaike, Information theory and an extension of the likelihood ratio principle, in: 2nd International Symposium on Information Theory, 1973, pp. $267-281$.

[42] C. Genest, K. Ghoudi, L.P. Rivest, A semiparametric estimation procedure of dependence parameters in multivariate families of distributions, Biometrika 82 (3) (1995) 543-552.

[43] E.C. Brechmann, U. Schepsmeier, Modeling dependence with C- and D-vine copulas: the R package CDVine, J. Stat. Softw. 52 (3) (2013) 1-27.

[44] I. Hobæk Haff, Comparison of estimators for pair-copula constructions, J. Multivar. Anal. 110 (2012) $91-105$.

[45] M.G. Kendall, Rank Correlation Methods, Hafner Publishing Co., New York, 1955.

[46] G. Allegri, S. Corradi, M. Marchetti, Stochastic analysis of the vibrations of an uncertain composite truss for space applications, Compos. Sci. Technol. 66 (2006) 273-282.

[47] Benedikt Gräler, Modelling skewed spatial random fields through the spatial vine copula, Spat. Stat. 10 (2014) $87-102$. 